\title{
Die Geburt der Kulturwissenschaft aus dem Geist des linguistic turn
}

\section{Überlegungen zu ihrem genealogischen Zusammenhang}

Hans-Martin Gauger zum 85. Geburtstag

Das Anliegen dieses Artikels besteht darin, die allgemeinen Implikationen dessen zu rekonstruieren, was der Terminus Kulturwissenschaft besagt. Ich werde mich deshalb sehr viel weniger um ihre durchaus vielfältigen Erscheinungsformen kümmern, sondern meine Aufmerksamkeit vor allem auf das richten, was ihre Varianten miteinander verbindet. Aus diesem Grund werde ich mich im Besonderen um eine Erklärung bemühen, warum es aufgrund der Entwicklung der Geisteswissenschaften - und vornehmlich derjenigen der Literaturwissenschaft(en) - zur Entstehung einer Kulturwissenschaft überhaupt kam.

Zunächst jedoch seien ein paar Bemerkungen zum vieldeutigen Begriff der Kulturwissenschaft selbst - genauer gesagt zu seinem Numerus - vorausgeschickt. ${ }^{1}$ Setzt man ihn in den Plural und spricht von Kulturwissenschaften, dann tritt dieser Terminus an die Stelle der traditionellen Bezeichnung Geisteswissenschaften. So trägt die Sektion 28 der Deutschen Nationalakademie Leopoldina, die neben den Theologien, der Jurisprudenz und einigen Sozialwissenschaften den gesamten Fächerkanon der ehemaligen Philosophischen Fakultäten versammelt, die Denomination Kulturwissenschaften. Im Fall eines solchen Begriffsgebrauchs in der Mehrzahl signalisiert der Terminus vor allem ein verändertes methodisches Bewußtsein. Die mit ihm verbundene Abgrenzung gegenüber der herkömmlichen Nomenklatur und gleichzeitige Annäherung von Kultur- und Naturwissenschaften hat nicht zuletzt eine methodische Affinität zwischen den traditionell eher zueinander in einen Gegensatz gestellten Wissenschaftskulturen zu signalisieren. Sie gibt im Besonderen zu verstehen, daß auch die wissenschaftliche Beschäftigung mit den Erzeugnissen des Menschen eines empirischen Zugangs zu ihnen bedarf.

Die herkömmliche Auffassung der Geisteswissenschaften und ihre Abgrenzung gegenüber den Naturwissenschaften läßt sich noch immer recht genau

1 Vgl. hierzu auch Hartmut Böhme: Was ist Kulturwissenschaft? (https://www.kuwi.europauni.de/de/lehrstuhl/sw/sw2/lehre/0809/Einfuehrung_in_die_Kulturwissenschaften/tutorium/ Literatur1/boehme.pdf).

Andreas Kablitz, Köln

Ә Open Access. () 2022 Andreas Kablitz, publiziert von De Gruyter. (cc) BY Dieses Werk ist lizenziert unter einer Creative Commons Namensnennung 4.0 International Lizenz. https://doi.org/10.1515/9783110667004-002 
mit Diltheys berühmt gewordener Unterscheidung zwischen zwei verschiedenen Formen des Zugriffs auf ihren jeweiligen Gegenstand der Erkenntnis, vermittels seiner Differenzierung zwischen >Erklären Den Geisteswissenschaften ist, folgt man den konzeptuellen Implikationen dieses deutlich hegelianisch konnotierten Begriffs im strengeren Sinne seiner Semantik, eine kategoriale Trennung zwischen dem Subjekt der Erkenntnis und seinem Gegenstand fremd. Wo von den entsprechenden Disziplinen in einem so verstandenen Sinn die Rede ist, begreift man ihr Geschäft wesentlich als eine Selbsterkundung des Geistes: Er hat - und weiß - sich in seinen verschiedenen kulturellen Entäußerungen stets wiederzuentdecken. (Wollte man ein theoretisches Modell für diese Selbstentäußerung des Geistes in der Tradition der Philosophie aufsuchen, so gibt es eine Reihe von Gemeinsamkeiten mit einem neuplatonischen Emanationsmodell zu bemerken.) Erkenntnis bedeutet unter diesen Voraussetzungen einen sympathetischen Nachvollzug fremden Erlebnisses, eben ein Verstehen (wobei der Wortsinn dieses Begriffs seiner geläufigen Bedeutung fast entgegenzustehen scheint):

Es gibt da keine hypothetischen Annahmen, welche dem Gegebenen etwas unterlegen. Denn das Verstehen dringt in die fremden Lebensäußerungen durch eine Transposition aus der Fülle eigener Erlebnisse. ${ }^{2}$

Es lohnt sich, einen Augenblick bei der durchaus suggestiven Wortwahl, die Dilthey zum Zweck dieser Abgrenzung der Geistes- gegenüber den Naturwissenschaften vornimmt, zu verweilen. Sie stellt sich bei genauerer Betrachtung nämlich als ein ziemlich genaues Gegenstück zur weithin verbreiteten naturwissenschaftlichen Skepsis gegenüber der Verläßlichkeit geisteswissenschaftlicher Forschungsergebnisse dar. Diltheys Formulierungen ziehen durch ihre Konnotationen im Gegenzug stattdessen die Vertrauenswürdigkeit naturwissenschaftlicher Erkenntnisse ein Stück weit in Zweifel. Die Suggestion einer solchen Skepsis beginnt in den zitierten Zeilen mit seiner Charakteristik des Verfahrens wissenschaftlicher Hypothesenbildung. Dilthey spricht von »hypothetischen Annahmen«. Schon diese Wortfügung ist sprechend. Hypothesen sind - aus dem Griechischen ins Deutsche übersetzt - nichts anderes als Unterstellungen, und eben diesen Wortsinn bringt der Relativsatz zum Ausdruck, der besagt, daß derlei Annahmen, die »dem Gegebenen etwas unterlegen« (Hervorhebung A. K.), auch wenn er das semantisch freilich nicht sonderlich entfernte - aber mit einem moralischen Unterton behaftete - Verb sunterstellen` vermeidet. Ebenso unterstreicht die Rede von einer - bloßen - Annahme, die die zur Unterlegung mutierte Un-

2 Wilhelm Dilthey: Der Aufbau der geschichtlichen Welt in den Geisteswissenschaften. Frankfurt a.M. 1970, S. 140. 
terstellung begleitet, den hypothetischen Charakter solcher Aussagen, indem sie sie ganz in die Perspektive des Erkenntnissubjekts hineinholt. Der stillschweigende Vorwurf, der daraus zu lesen ist, steckt mithin in einer Zuschreibung von Äußerlichkeit (und damit Unverbindlichkeit), die jeglicher Hypothese gegenüber dem, was sie erklären soll, zu eignen scheint. Im Unterschied dazu kennzeichnet Dilthey das Verstehen gerade durch die Betonung seines Eindringens in seinen Gegenstand. Bis in die Details seiner raffinierten Formulierungen arbeitet Dilthey an einer unausdrücklichen Abwertung naturwissenschaflticher Aussagen. Ihrem Unverbindlichkeit signalisierenden Plural (»Es gibt da keine hypothetischen Annahmen«, was in etwa besagt: ১Es gibt nicht irgendwelche Annahmen`), stellt er die Zuverlässigkeit eines mit dem bestimmten Artikel versehenen Verstehens gegenüber, bei dem der Vorgang der damit bezeichneten Erkenntnis vom Subjekt dieser Erkenntnis gar nicht mehr zu unterscheiden zu sein scheint. Denn logisch deutet die Erwähnung »eigener Erlebnisse« auf dieses Subjekt, grammatisch aber beziehen sie sich auf das Verstehen; und diese Überblendung von Logik und Grammatik läßt das Ich in seinem Verständnis des Anderen gleichsam aufgehen.

Eine Stütze findet Diltheys heimliche Absicherung des solchermaßen geschilderten kognitiven Vorgangs zudem im Rückgriff auf das Leben als dem allen gemeinsamen Urgrund jeglichen Verstehens. Dabei sollte man nicht übersehen, daß das Leben zugleich in Gestalt von Lebensäußerungen in Erscheinung tritt. Es arbeitet dem Verstehen schon vor, ja scheint im Verständnis durch einen Anderen nachgerade seine Bestimmung zu finden; denn Äußerungen sind stets an jemanden gerichtet. In Diltheys Rekurs auf das Leben steckt darüber hinaus eine implizite Beschreibung des Mechanismus, wie das Verstehen als ein "Sich in die Position des Anderen Begebens « funktionieren soll (und kann). Es ist im Grunde die Fülle eigener Erlebnisse, also letztlich die Fülle des Lebens selbst, die für eine solche »Transposition « die erforderlichen Voraussetzungen schafft. Im Reichtum des Lebens, in der Vielfalt seiner Manifestationen, scheint sich die Distanz zwischen dem Eigenen und dem Fremden, die ja auch Diltheys Worte keineswegs leugnen, überwinden zu lassen, als hielte dieses Leben eine Mannigfaltigkeit bereit, in der stets ein Ort der Begegnung mit dem Andersartigen zu finden wäre. Vermittels seiner zweifellos äußerst geschickt arrangierten Formulierungen spielt Dilthey auf die beschriebene Weise ein in den Wurzeln des Lebens selbst verankertes Verstehen gegen eine Erklärung aus, die an der bloßen Oberfläche verbleibt, ja mit von außen kommenden Unterstellungen operiert, die auch in der unschuldigeren sprachlichen Variante von Unterlegungen ihren pejorativen Duktus kaum einbüßen. 
Die Literaturwissenschaftler kennen die Konsequenzen eines solchen methodischen Programms zur Genüge. Seine reinste Ausprägung hat es in ihrer Disziplin die diesen Namen an dieser Stelle gewissermaßen programmatisch unterläuft - vermutlich im Postulat der Einfühlung - das man eine Methode nicht recht nennen mag - gefunden. Für lange Zeit schien es einen privilegierten Zugang zum Text der Lyrik zu garantieren. Seinen Kern bildet die Vorstellung von einer Aufhebung der epistemologischen Grenze zwischen Erkenntnissubjekt und -objekt, die auf eine Verschmelzung zwischen dem Produzenten und dem Rezipienten des Textes zielt. Mit seiner ostentativ antirationalen Stoßrichtung verspricht schon der Begriff der Einfühlung, durch emotionale Einstimmung die Grenzen überwinden zu können, die die Vernunft - der Epistemologie der Moderne zufolge - zwischen dem Ich und seiner Welt unweigerlich errichtet. Die solchermaßen verstandene Einfühlung bleibt übrigens keineswegs auf Artefakte begrenzt. Im Fall der Ereignisgeschichte etwa nimmt sie die Gestalt einer imaginären Verwandlung in den Akteur des historischen Geschehens an.

Ein entsprechendes Verfahren, das Objekterkenntnis mit dem imaginären Nachvollzug fremden Verhaltens identifiziert, ist uns allerdings längst fragwürdig geworden.

Im Grunde hat schon der Historismus gehörige Zweifel daran geweckt, daß sich die kulturelle Welt so bruchlos als die Selbstentfaltung des einen und mit sich selbst identischen Geistes über die Zeiten hinweg begreifen läßt - ganz zu schweigen von der Skepsis, die ein ethnologischer Blick auf die Kultur gegenüber einer solchen Konzeption zur Folge haben muß. ${ }^{3}$ Und auch die Verschiebung der Möglichkeiten, Einsicht durch Einfühlung zu gewinnen, auf eine emotionale Basis hat mit dieser Verwandlung ihrer Voraussetzungen das Vertrauen in die Leistungsfähigkeit unvermittelter Erkenntnis kaum dauerhaft erhöhen können.

Die Relativierung der von Dilthey formulierten kognitiven Differenz zwischen Geistes- und Naturwissenschaften ist bekanntlich von verschiedenen Seiten betrieben worden. Gewirkt in diesem Sinn hat nicht zuletzt die wissenschaftstheoretische Diskussion, die - etwa im Gefolge Karl Poppers - die Theorieabhängigkeit jeder Empirie herausgestellt hat. Ebenso mehren sich in den Geisteswissenschaften selbst vor allem in der zweiten Hälfte des 20. Jahr-

3 Wenn ich an dieser Stelle so vorsichtig formuliere, dann deshalb, weil auch ethnologische Konzeptionen keineswegs zwangsläufig eine gleichsam kommunitaristische Methodologie mit sich führen müssen. Eines der prominentesten Gegenbeispiele im 20. Jahrhundert bieten zweifellos Claude Lévi-Strauss' Mythologiques, die - aller strukturalistischen Ausdehnung zum Trotz - einen ausgesprochen hegelianischen Geist atmen. 
hunderts kritische Stimmen gegen Diltheys methodologisches Programm, am entschiedensten vielleicht in der Konsequenz des Strukturalismus.

Aber auch innerhalb der Entwicklung der Hermeneutik läßt sich eine kritische Revision von Diltheys Position beobachten. Hans-Georg Gadamers lange einflußreiches Buch Wahrheit und Methode wird man in diesem Sinn als eine Umbesetzung des Verhältnisses zwischen dem Erkenntnissubjekt und seinem Gegenstand innerhalb der Koordinaten von Diltheys Epistemologie beschreiben können. Gadamers Begriff der Tradition nimmt sich nämlich wie eine Instanz der Vermittlung zwischen den beiden an jedem Erkenntnisprozeß beteiligten Entitäten aus: Tradition ist bei ihm zum einen das, was sie im wörtlichen Sinn bedeutet: Sie ist Weitergabe - Weitergabe dessen, was sich für eine Überlieferung lohnt (wobei er einen untrüglichen Sinn für eine stets gelingende Identifizierung des dafür Tauglichen vorauszusetzen und sogar die Selbstbezüglichkeit von solchen Verstetigungen auszuschließen scheint. Aber tradiert man nicht auch deshalb etwas, weil man es immer schon tradiert hat, obwohl der Gegenstand dieser Weitergabe inzwischen längst an Belang eingebüßt hat?).

Tradition aber meint zum anderen nicht allein den Vorgang, den das Verb tradere namhaft macht; sie schließt ebenso das Tradierte ein. Dieser doppelte Bezug des Traditionsbegriffs macht sie im gleichen Zug zu einem Instrument wie zu dem Ort der Vermittlung zwischen dem Erkenntnissubjekt und seinem Objekt. Deren Zusammenhang wird nicht mehr, wie bei Dilthey, durch eine imaginäre Verwandlung des Erkennenden in den Urheber des zu erkennenden Gegenstands bewerkstelligt. Sie wird vielmehr durch die Modalitäten einer Aneignung bewirkt. Mit einer solchen Aneignung aber kehrt sich die Perspektive gegenüber dem Verstehen um und markiert den Anteil des Subjekts an der Weitergabe des zu Tradierenden. Dabei ist der Begriff der Tradition selbst dazu geeignet, dieses Moment der Appropriation zu verdecken, erweckt er doch den Eindruck, als beschränke sich die Weitergabe durch das Subjekt auf den bloßen Vorgang eines Weiterreichens, das das Weitergegebene intakt beläßt. De facto aber setzt Gadamers Tradition die jeweilige Aneignung des Weiterzugebenden voraus, die eine stets erneute Anpassung an sich beständig wandelnde Voraussetzungen seiner Rezeption mit sich führt. Hier gibt es nicht mehr einen gemeinsamen Fundus von Erfahrungen, den die Fülle des Lebens bei Dilthey als Ermöglichungsgrund eines gelingenden Verstehens voraussetzt. Gadamer sieht sich im Gegenteil gerade mit dem Problem eines unablässigen Wandels von Verstehensvoraussetzungen konfrontiert. Deshalb stellt er auch die Prozeßhaftigkeit der Tradition heraus, die sie vom Vorgang des Verstehens bei Dilthey unterscheidet. Bei ihm spielt ein jedes Verstehen allein zwischen dem einen Subjekt und seiner Erkenntnis des Gegenstands. Jedes Verstehen setzt deshalb kein vorgängiges Verstehen desselben Objekts durch ein anderes Subjekt dieser Erkenntnis voraus. Alle Tradition spielt 
stattdessen immer schon in einer Serie von Weitergaben, die einer Folge von Veränderungen gleichkommt, die durch die sich ändernden Bedingungen des Verstehens erzwungen werden. Diltheys Eindringen in das Objekt des Verstehens ist deshalb in Wahrheit und Methode die Angleichung des zu verstehenden Objekts an die sich wandelnden Lebensumstände des Verstehenden gegenübergestellt.

Die Vermittlungsleistung der Tradition aber verlangt ihre Preise; denn sie verwischt die Grenze zwischen Aneignung und Angeeignetem. Da die Weitergabe des Tradierten zur Aufrechterhaltung der Tradition stets nach den Maßgaben des Subjekts dieser Weitergabe erfolgt, ändert sich Zug um Zug auch das Weitergegebene. (Dabei steckt in jedem Akt der Tradition im Grunde ein leises Paradox. Denn er betreibt die Aneignung in der Gegenwart im Blick auf die Weitergabe an eine Zukunft, in der sich die Bedingungen des Verständnisses - und möglicherweise sogar der Belang dessen, was sich überhaupt verstehen läßt bereits wieder geändert haben werden. Alle Tradition kommt insoweit einem ungedeckten Wechsel auf die Zukunft gleich.) Die Überwindung der epistemologischen Grenze zwischen dem Erkenntnissubjekt und seinem Gegenstand ist in Gadamers Wahrheit und Methode um den Preis der Relativierung ihrer differentiellen Identität erkauft. Verständnis aber droht in der Konsequenz dieser Grenzverwischung letztlich durch eine Usurpation ersetzt zu werden, die jegliches Urteil über die Angemessenheit einer Aneignung in Zweifel zieht. ${ }^{4}$

So wird man fairerweise konzedieren müssen, daß sich der geläufige Gebrauch des Begriffs Geisteswissenschaften längst von den theoretischen Implikationen gelöst hat, die sein Wortsinn transportiert, und im Grunde kaum mehr betreibt, als den Gegenstandsbereich der mit ihm bezeichneten Disziplinen abzugrenzen. ${ }^{5}$

\footnotetext{
4 Im Grunde fragt sich natürlich bereits im Blick auf Diltheys `Einfühlung`, ob sie nicht ihrerseits ein bloßes Programm bleibt, das de facto stets in das Postulat eines Nachvollzugs fremden Erlebnisses mündet. Da es an jedweder Kontrolle über den Erfolg der Einfühlung fehlt, bleibt nur die Behauptung ihres Gelingens übrig, die auf einem subjektiven Eindruck fußt.

5 Auch dies gelingt freilich nicht in der wünschenswerten Trennschärfe. Fragen der Zuordnung bleiben im Blick auf bestimmte Disziplinen vor allem für das Verhältnis zu den Sozialwissenschaften offen. Solche Schwierigkeiten scheint der Begriff der Kulturwissenschaften vermeiden zu können. Aber ob sich die Ökonomen, die äußerst bedacht auf die empirische Basis ihrer Untersuchungen sind, sonderlich wohl in einem solchen Verbund fühlen, steht doch recht entschieden dahin. (Ganz abgesehen davon, ob sich in strengem Sinn dann nicht auch die Naturwissenschaften als Kulturwissenschaften zu begreifen hätten; denn schließlich ist auch ihr Konzept von Natur stets ein Produkt kultureller [Vor-]Entscheidungen.) Wie man sieht, eignet der Nomenklatur der Wissenschaften stets ein Moment des Aleatorischen. Völlige Kohärenz scheint hier, mit welcher Begrifflichkeit auch immer, kaum herzustellen zu sein.
} 
In diesem Sinn verstanden, bildet das Aufkommen des Begriffs einer Kulturwissenschaft weit eher ein Symptom eines generellen Prozesses der Transformation der Geisteswissenschaften im 20. Jahrhundert, statt eine Revolution anzuzeigen und macht vor allem ein anti-hermeneutisches Konzept geltend. Ihr Anliegen ist es, das im Begriff der Geisteswissenschaften benannte Programm einer Selbsterkundung des menschlichen Geistes zu verabschieden. An die Stelle einer Selbstdeutung habe Objekterklärung zu treten. Das alte diltheysche Begriffspaar von Verstehen und Erklären erfaßt auch diesen Unterschied recht präzise. Nur dient es nun nicht mehr zur systematischen Differenzierung zwischen Geistes- und Naturwissenschaften, sondern zu einer historischen Unterscheidung von Geistes- und Kulturwissenschaften.

Ich will mich auf die Beziehung zwischen diesen beiden Begriffen hier nicht weitergehend einlassen. Allerdings scheinen sie mir weit weniger geeignet, jene methodologischen Differenzen angemessen zum Ausdruck zu bringen, für deren Gegenüberstellung Dilthey sie gewählt hat. Sie stellen, vom Wortsinn der beiden Begriffe her gedacht, ein kognitives Verfahren (`verstehen`), einer kommunikativen Praxis (`erklären`) gegenüber. Doch beides korreliert keineswegs eindeutig mit den von Dilthey Natur- und Geisteswissenschaften jeweils verordneten methodischen Programmen. Denn auch eine Deutung, sagen wir eines Gedichtes, ist eine Erklärung desselben, wie sich umgekehrt ein Kausalmechanismus eines natürlichen Vorgangs durchaus auch verstehen läßt. Der entscheidende Unter-

Ich beschränke mich darum bei meinen Überlegungen auch auf das Verhältnis zwischen den Begriffen »Kultur-« und »Geisteswissenschaften« und den mit ihnen verbundenen methodischen Implikationen. Dabei bleiben viele Fragen offen. So gälte es etwa zu klären, warum die Sozialwissenschaften, die sich mit überindividuellen kulturellen Formationen, aber gleichwohl kulturellen Hervorbringungen des Menschen beschäftigen, eine größere Affinität zu empirischen Verfahren als die hermeneutisch operierenden Geisteswissenschaften zu besitzen scheinen. Wären kollektive Institutionen, die sich nicht mehr einem letztlich dialogischen Verhältnis zwischen zwei Individuen subsumieren lassen, grundsätzlich für ein anderes methodisches Prozedere disponiert? Die Geschichte der Sozialwissenschaften spricht dagegen; denn eine empirisch basierte Soziologie ist, unter einem historischen Gesichtspunkt betrachtet, keineswegs alternativlos. Und wie verhält sich die vor allem im Französischen gebräuchliche, aber auch hierzulande keineswegs unvertraute Terminologie der sciences humaines oder $\mathrm{Hu}$ manwissenschaften zu den hier aufgeworfenen Fragen? Sie weist gleichermaßen ihre Unschärfen auf. Denn vermittels ihres Gegenstands bestimmt, werden ihre Grenzen dort undeutlich, wo der Mensch selbst ins Zentrum des Erkenntnisses rückt. In welchem Maße wird man die Medizin zu ihrem Geltungsbereich ziehen müssen, und verläuft nicht gerade mitten durch die Psychologie eine solche Grenze? (Wie stünde es im Übrigen um die angelsächsischen humanities, die sich in jedenfalls terminologisch irritierender Weise als arts and humanities präsentieren und damit einen Wissenschaftsbereich bezeichnen so wie eine kulturelle Praxis, die zu dessen privilegierten Erkenntnisobjekten zählen darf.) 
schied scheint sich deshalb zwischen einer Deutung und einer Messung abzuspielen, der maßgeblichen Einfluß auf die Zuverlässigkeit ihrer Ergebnisse, im Besonderen ihrer Regelmäßigkeitsannahmen hat. Verstehen wie Erklären, Deuten wie Messen aber stellen einen Rationalitätsgewinn dar. Und eben darin, in ihrer Rationalisierungsleistung, scheinen mir die Gemeinsamkeiten der verschiedenen wissenschaftlichen Verfahren zu bestehen.

Neben den Kulturwissenschaften gibt es die Kulturwissenschaft im Singular. Sie versteht sich zunächst als eine Überwindung der überkommenen disziplinären Aufteilung wissenschaftlicher Beschäftigung mit der Kultur in eine (zudem beständig zunehmende) Reihe verschiedener Fächer, deren erkenntnisbehindernde Grenzen sie beklagt. Indessen läßt sich in diesem Zusammenhang eine institutionelle Beobachtung machen, die in konzeptueller Hinsicht äußerst lehrreich ist. Wo sich nämlich ein Fach `Kulturwissenschaft $\triangleleft$ mit eigenem Institut und eigenem Studiengang etabliert, befindet es sich vielfach in einem disziplinären Verbund mit vorzugsweise der Literaturwissenschaft und siedelt sich in Deutschland gern im Umfeld der Germanistik an.

Damit sind wir auch schon beim Kernanliegen dieses Beitrags angekommen. Ich möchte zu erklären versuchen, warum gerade die Philologien, die solche immer weniger sein möchten, ein vorrangiges Interesse an einer Kulturwissenschaft bekunden. Wenn denn die Überwindung tradierter Disziplinengrenzen bei der akademischen Erschließung der Kultur die eigentliche (oder zumindest primäre) Zielsetzung ihrer Begründung bildet (oder bildete), so wäre ein verhältnismäßig gleichmäßig verteilter Enthusiasmus für sie in den dereinst geisteswissenschaftlichen Fächern zu erwarten. Doch dem ist nicht so. Allen voran die Philologien und im Besonderen die verschiedenen Literaturwissenschaften haben sich zu inzwischen erheblichen disziplinären Anteilen einer kulturwissenschaftlichen Ausrichtung verschrieben, die sich längst auch institutionell etabliert hat und etwa in der Denomination von Professuren zum Ausdruck kommt. Warum aber ist das so?

Um meine zentrale These schon an dieser Stelle vorwegzunehmen: Die Ursache der kulturwissenschaftlichen Wende in der Literaturwissenschaft scheint mir eine Folge des sog. linguistic turns darzustellen; ${ }^{6}$ und die daraus resultierende Kulturwissenschaft verwaltet in der Konsequenz ihres konzeptuellen Ursprungs das durchaus zwiespältige Erbe der mit diesem Begriff bezeichneten Veränderungen in den

6 Das Aufkommen einer Kulturwissenschaft scheint mir insofern eher rezente Ursachen zu haben, Böhme: Was ist Kulturwissenschaft? (s. Anm. 1) verfolgt stattdessen die longue durée einer Kulturwissenschaft, deren Wurzeln er in einer semantischen Entwicklung des Kulturbegriffs angelegt sieht, den er seit der griechischen und römischen Antike zu rekonstruieren unternimmt. 
Grundlagen unseres Denkens im 20. Jahrhundert ausgesprochen schlüssig. (Nur um einem Mißverständnis vorzubeugen: Ich beziehe mich mit meiner These ausschließlich auf jene Formen von Kulturwissenschaft, die eine erkennbare konzeptuelle Veränderung gegenüber der herkömmlichen Literaturwissenschaft mit sich führen. Nicht selten wird dieses Etikett durchaus überkommenen Verfahren aufgeklebt. Dies gilt etwa für motivgeschichtlich angelegte Arbeiten zur gender-Thematik oder welchem kulturwissenschaftlich relevanten Gegenstand auch immer. Und dies gilt ebenso für Applikationen von Kulturtheorien auf literarische Texte - also etwa der horrend mißverstandenen thick description von Clifford Geertz. ${ }^{7}$ Eine solche Praxis schreibt nichts anderes als ein wohletabliertes hermeneutisches Verfahren fort, das freilich als ein solches nur zu gern verkannt wird, weil das thematische Interesse für die Kultur die Sicht für das methodische Procedere zu verstellen scheint. Doch ob man eine Kulturtheorie, Freuds Psychoanalyse oder Foucaults Diskursarchäologie auf einen Text in hermeneutischer Absicht anwendet, macht, wissenschaftssystematisch betrachtet, keinerlei Unterschied).

Worin aber besteht die angedeutete fundamentale Ambivalenz im zwiespältigen Erbe des linguistic turns? ${ }^{8}$

Sie läßt sich gleichsam als ein Pyrrhussieg der Sprache beschreiben. Wenn denn gilt, daß unser Zugang zur Wirklichkeit wesentlich, wo nicht ausschließlich, von der Sprache bestimmt ist, dann erfährt die Sprache zweifellos eine beträchtliche, ja immense Bedeutungssteigerung. Doch ein solcher Relevanzgewinn hat seine alles andere als nebensächlichen Preise. Denn er ist erkauft um einen Substanzverlust der Sprache. Wenn es sich nämlich so verhält, daß sie maßgeblich den Zugriff auf die Welt regelt, wer garantiert dann, daß die Sprache diese Welt nicht allererst formiert, wo nicht konstituiert - und damit unwiederbringlich das verspielt, was man traditionell als die Wirklichkeit verstanden hat? In den Prämissen des linguistic turns steckt insofern der Keim ontologischer Skepsis. Hier hat die gar nicht mehr enden wollende, ja sich stetig fortzeugende Rede von der Selbstreferenz der Sprache - einer Autoreflexivität, die in der Literatur

7 Dieses fundamentale Mißverständnis seines Ansatzes gründet zu erheblichen Teilen darauf, daß man thick description für einen un-, wo nicht anti-hermeneutischen Zugriff auf literarische Texte in Anspruch hat nehmen wollen, während diese Formel bei Geertz selbst als Gegenbegriff zu einer thin description gerade den hermeneutischen Zusatz gegenüber einer bloßen Beschreibung markiert. Schon der Wortlaut der einschlägigen Kapitelüberschrift Thick Description. Toward an Interpretive Theory of Culture (Clifford Geertz: The Interpretation of Cultures. Selected Essays. With a foreword by Robert Darnton. New York 2017, S. 3) sollte in dieser Hinsicht allerdings hellhörig machen (vgl. hierzu des Näheren Kablitz: Die Sprachlichkeit des Textes. Vom Nutzen und Nachteil seiner Metaphorisierung und von deren Ursachen. In: POETICA 48 (2016), S. 169-200).

8 Zum Verständnis dieses Begriffs siehe unten Anm. 19. 
ihre Selbstoffenbarung (wie -vollendung) betreibe - einen wesentlichen Ursprung. Denn wo die Sprache auf keine ihr äußerliche Größe verweisen kann, bleibt ihr letztlich nur noch die Selbstbezüglichkeit.

Die Bestimmung literarischer Rede zur Aufklärungsarbeit, ihre Beanspruchung für eine Aufdeckung der realistischen Illusionen, die die Sprache produziert, ist vor allem ein Anliegen Paul de Mans. Bei ihm läßt sich sehr präzise verfolgen, wie das Prinzip der Selbstreflexivität mit dem Postulat ontologischer Grundlosigkeit der Sprache einhergeht.

Seinen Ausgangspunkt bildet der Befund einer grundsätzlichen Unzulänglichkeit der Grammatik für die Dekodierung sprachlicher Äußerungen, die ihr eine nicht aufzulösende Marge der Unbestimmtheit (indetermination) belasse:

[...] the grammatical decoding of a text leaves a residue of indetermination that has to be, but cannot be, resolved by grammatical means, however extensively conceived. ${ }^{9}$

Diese Unbestimmtheit, alias Polysemie, aller Rede trete nun in besonderer Weise in der Literatur zutage:

[...] literature is not a transparent message in which it can be taken for granted that the distinction between the message and the means of communication is clearly established. ${ }^{10}$

Es ist eine Beschreibung literarischer Selbstreflexivität, die auch mit diesen Worten stattfindet. Denn die dabei diagnostizierte mangelnde Unterscheidbarkeit von Botschaft und Medium besagt genau dies: Wenn die Botschaft der Rede sich nicht recht von den medialen Verfahren ihrer Produktion separieren läßt, dann wird sie unweigerlich selbstbezüglich.

Für die weiteren Folgerungen, die de Man daraus ableiten wird, ist es nicht unerheblich, daß sich sein Konzept literarischer Selbstbezüglichkeit in einem wesentlichen Punkt von Roman Jakobsons berühmter Definition poetischer Autoreflexivität, die die Karriere dieses Begriffs in der Literaturwissenschaft weithin begründet hat, deutlich unterscheidet. In seinem epochemachenden Aufsatz Linguistics and Poetics bestimmt er die poetic function der Sprache bekanntlich wie folgt:

The set (Einstellung) toward the message as such, focus on the message as such, is the POETIC function of language. ${ }^{11}$

9 Paul de Man: The Resistance to Theory. In: Ders.: The Resistance to Theory. Foreword by Wlad Godzich. Manchester 1986, S. 3-20, hier S. 15.

10 Ebd., S. 15.

11 Roman Jakobson: Linguistics and Poetics. In: Style and Language. Hg. von Thomas A. Sebeok. Cambridge 1960, S. 350-377, hier S. 356. 
Während Jakobson die Selbstbezüglichkeit der Botschaft zum fundierenden Merkmal poetischer Autoreflexivität erklärt, ist es bei de Man die Unmöglichkeit einer Unterscheidung zwischen Botschaft und Medium. Dieser Unterschied ist durchaus folgenreich. Der von de Man reklamierte Verweis literarischer Rede auf das Medium als solches amalgamiert nämlich zwei der Sprachfunktionen, zwischen denen Jakobson deutlich unterschieden hatte: die poetische und die metasprachliche Funktion. Wenn de Man sie hingegen bis zur Ununterscheidbarkeit miteinander vermischt, so scheint ihre Verquickung ihm einen bestimmten und offensichtlich durchaus erwünschten argumentativen Vorteil zu verschaffen: Sie bereitet seiner ontologischen Inanspruchnahme der Literatur das Terrain:

Literature is fiction not because it somehow refuses to acknowledge >reality`, but because it is not a priori certain that language functions according to principles which are those, or which are like those, of the phenomenal world. ${ }^{12}$

Genau dies benennt die ontologische Wende der Argumentation: Wenn Literatur fiktiv ist, dann deshalb, weil nicht gewiß sein kann, daß die Prinzipien der Sprache denjenigen der Wirklichkeit korrespondieren.

Wir sollten nicht übersehen, welcher sprachlichen Mittel es bedarf, um diese Wende auf den Weg zu bringen. Sie stecken in de Mans Darstellung oder besser: in seiner Aufbereitung - des üblichen Verständnisses von literarischer Fiktion. Denn er sagt ja nicht etwa, diese Auffassung bestünde darin, daß Literatur etwas Nicht-Wirkliches zum Gegenstand habe. Vielmehr erscheint das betreffende Verständnis bei ihm als eine Verweigerung der Anerkenntnis von Realität (it somehow refuses to acknowledge sreality). Auf diese Weise wird eine zeichenhafte Relation in eine kognitive Beziehung, präziser noch gesagt: in eine ontologische Haltung umgedeutet. Der Verweis auf etwas nicht Faktisches wandelt sich unter der Hand zu einer Zurückweisung der Wirklichkeit, und diese Transformation ermöglicht es de Man im Anschluß, sein Konzept literarischer Fiktion im Gegenzug als eine Aufdeckung der Illusionen sprachlich generierter Wirklichkeitsvorstellungen zu präsentieren.

Um sein Ziel erreichen zu können, um die semiotische Selbstreflexivität in ontologische Aufklärung umwidmen zu können, braucht de Man mithin einige sprachliche Winkelzüge, die die Voraussetzungen dafür erst schaffen, aber dessen Plausibilität nicht unbedingt erhöhen. Denn eben dies ist sein Vorhaben: Die Literatur soll zum Herold einer ontologischen Grundlosigkeit avancieren, die andernorts - in anthropologischer Zurichtung - ein noch drastischeres Profil gewinnt:

12 De Man: Resistance to Theory (s. Anm. 9), S. 11. 
Here the human self has experienced the void within itself and the invented fiction, far from filling the void, asserts itself as pure nothingness, our nothingness stated and restated by a subject, that is the agent of its own instability. ${ }^{13}$

Steht der ontologische Vorbehalt in Resistance to Theory noch im Zeichen der Ungewißheit über eine ontologische Grundlage sprachlicher Verfahren, so scheint hier die Gewißheit aller metaphysischen Gewißheit ganz ungebrochen zu sein.

Mobilisiert Paul de Man die Selbstreflexivität poetischer Rede also für einen Feldzug gegen alle der Sprache unterstellten ontologischen Ansprüche, so scheint Jakobsons Bestimmung der poetischen Funktion frei von derlei anti-metaphysischen Prätentionen zu sein. Indessen scheint sich in seinem Fall die Autoreflexivität der Literatur im Zeichen einer anderen Kategorie zu formieren: Sie nimmt sich wie eine semiotische Reinterpretation der aus der philosophischen Ästhetik stammenden Kategorie der Autonomie aus - einer ästhetiktheoretischen Variante der Kategorie der Freiheit. Sie hält gleichsam die Wirklichkeit auf Distanz zum Zweck einer ästhetischen Selbstverwirklichung des Subjekts (der Kunst), das sich durch sie von den Notwendigkeiten der Natur zu lösen vermag. Paul de Man also überführt mit seiner Umwidmung der Selbstbezüglichkeit der Literatur ästhetische Distanzierung von der Wirklichkeit in eine kognitive Funktion: in das aufklärerische Programm einer Entlarvung der Nichtigkeit aller Wirklichkeit. Allerdings wird man vielleicht nicht fehlgehen, auch diese ontologische Umdeutung poetischer Selbstbezüglichkeit als eine Vollendung ästhetischer Freiheitsansprüche des Menschen zu begreifen.

Aus disziplinengeschichtlicher Sicht betrachtet, bildet die Kulturwissenschaft m. E. übrigens so etwas wie das Gegenstück zur Stilistik der ersten Hälfte des 20. Jahrhunderts. Bei Auerbach wie bei Spitzer, um zwei der Großen meines eigenen Faches zu nennen, bildet die Besonderheit, die sich in einem bestimmten Stil des Sprachgebrauchs manifestiert, die Abweichung vom Üblichen. Der écart, wie die französische stiltheoretische Bezeichnung lautet, liefert den Schlüssel zum Verständnis des Textes. Er gewährt Einblick in die Weltanschauung, die ihm zugrunde liegt, ${ }^{14}$ wie in die Umstände, die ihn hervorgebracht haben. ${ }^{15}$ Bezeichnenderweise - und ganz unaristotelisch, aber zugleich zutiefst

13 Paul de Man: Blindness and Insight. Essays in the Rhetoric of Contemporary Criticism. Introduction by Wlad Godzich. Minneapolis ${ }^{2} 1983$, S. 18.

14 Das Paradebeispiel dafür bietet noch immer Erich Auerbach: Mimesis. Dargestellte Wirklichkeit in der abendländischen Literatur. Bern 1946.

15 Siehe etwa Spitzers Studie zu Rabelais, die aus seiner Kölner Antrittsvorlesung von 1930 hervorgegangen ist und in der er aus dem Stil dieses Autors die Merkmale von dessen Kreativität herausliest: Leo Spitzer: Zur Auffassung Rabelais'. In: Ders.: Romanische Stil- und Literaturstudien I. Marburg 1931, S. 109-134. 
romantisch - eröffnet dort das Individuelle und nicht etwa das Allgemeine den Zugang zur eigentlichen Wirklichkeit. Aber eben zu ihr - und nicht etwa nur zu ihrer bloß sprachlich hervorgebrachten Schimäre - führt die signifikante, weil deviante Sprache.

Es wäre wissenschaftshistorisch womöglich ein aussichts- und aufschlußreiches Unternehmen, der Frage nachzugehen, ob ein solches Verständnis des vom Üblichen abweichenden Sprachgebrauchs als eines Schlüssels zur Welt womöglich schon jener generellen Sprachskepsis vorarbeitet, die sich dann im späteren 20. Jahrhundert der Philologien - und vor allem ihres literaturwissenschaftlichen Teils - bemächtigen wird. (Es liegt auf der Hand, daß dieser Spur des Näheren nachzugehen sich im Rahmen des vorliegenden Artikels verbietet.)

Eine solche Untersuchung hätte selbstredend über die Stilistik hinaus weitere theoretische Modelle einzubeziehen, die Momente der Negativität zum zentralen Merkmal literarischer Rede machen, im Besonderen das von den Russischen Formalisten entwickelte Konzept der Verfremdung. Im Rahmen ihrer Theoriebildung erscheint die Verfremdung wesentlich als ein literarisches Verfahren (приём), wie der terminus technicus lautet: als ein technisches Mittel der Aufmerksamkeitslenkung, die das Gemachtsein des literarischen Werkes als dessen Essenz zu erkennen geben soll:

Ziel der Kunst ist es, ein Empfinden des Gegenstands zu vermitteln, als Sehen, und nicht als Wiedererkennen; das Verfahren der Kunst ist das Verfahren der >Verfremdung ‘ der Dinge und das Verfahren der erschwerten Form, ein Verfahren, das die Schwierigkeit und Länge der Wahrnehmung steigert, denn der Wahrnehmungsprozeß ist in der Kunst Selbstzweck und muß verlängert werden; die Kunst ist ein Mittel, das Machen einer Sache zu erleben; das Gemachte hingegen ist in der Kunst unwichtig. ${ }^{16}$

So überraschend die Übereinstimmung auf den ersten Blick erscheinen mag, hält man die betreffenden Paradigmen jedoch gemeinhin für Antipoden, aber in dieser Betonung des Prozesses der Wahrnehmung, die zu erwecken das Ziel der Rezeption eines literarischen Werkes darstellt und die den Sinn für das Gemachtsein des literarischen Werkes befördern soll, steckt durchaus eine Gemeinsamkeit mit Diltheys Hermeneutik, die das Erlebnis des Kunstwerks als

16 Viktor Šklovskij: Die Kunst als Verfahren. In: Texte der russischen Formalisten, Band I, Texte zur allgemeinen Literaturtheorie und zur Theorie der Prosa. Hg. von Jurij Striedter. München 1969, S. 2-35, hier S. 15. »Целью искусства является дать ощущение вещи как видение, а не как узнавание; приемом искусства является прием «остранения» вещей и прием затрудненной формы, увеличивающий трудность и долготу восприятия, так как воспринимательный процесс в искусстве самоцелен и должен быть продлен; искусство есть способ пережить деланье вещи, а сделанное в искусстве не важно« (Viktor Shklovskii, »Iskustvo, kak priem«. In: O teorii prozy. Moskau 1929, S. 5-23, hier S. 13). 
einen Nachvollzug der Umstände seiner Entstehung begreifen lehrt: Hier wie dort ist eine Marginalisierung des Produkts - des Werkes - zugunsten seiner Produktion zu beobachten.

Im Unterschied zu Dilthey aber fassen die Russischen Formalisten diese Akzentuierung der Produktion in Kategorien der Negativität (während Dilthey sie im transcendentale des Lebens und seiner Fülle aufzuheben versucht). Erkenntnisfördernd ist für die Formalisten hingegen die Abweichung selbst - die Differenz gegenüber dem Vertrauten, die auf keine Fixierung dieses Unterschieds hinausläuft. Denn die Kunst ist für sie als solche eine Einrichtung permanenter Verfremdung, die insofern nur den unabschließbaren Prozeß der Verfremdung selbst zur Voraussetzung wie zum Ziel hat. Diese Verfremdung aber zielt über die Kunst hinaus, betreibt sie doch die Verfremdung der Dinge. Im ästhetiktheoretischen Konzept der Verfremdung steckt insofern insgeheim eine negative Ontologie, die von poststrukturaler Sprach- wie Literaturtheorie letztlich nicht sehr weit entfernt ist.

Es lohnt, für einen Augenblick die epistemologischen Voraussetzungen des linguistic turns selbst etwas näher zu bedenken. Denn sie können helfen, die Bedeutung zu verstehen, die er für das Verständnis der Sprache im Denken der Moderne besitzt. Im Grunde bringt er nämlich etwas zu Bewußtsein, was je schon im Nachdenken über das Denken, anders gesagt, in der Philosophie angelegt war. Auch Platons Ideen basieren im Grunde ja auf Begriffen - auf Begriffen, die durch die Aufnahme ins Reich der Ideen, gleichsam einer metaphysischen Himmelfahrt, mit ontologischer Substanz ausgestattet werden. Aber geboren sind sie aus der Sprache, an deren Leitfaden sich die Selbstreflexion des Denkens entwickelt.

Seinem Selbstverständnis nach erklärt das Denken die Sprache zu seinem Instrument und begreift sie als ein bloßes Hilfsmittel seiner Selbstverständigung. Der Logos selbst ist insofern als sprachunabhängig gedacht; er bedient sich der Sprache nur für diese Selbstverständigung wie gleichermaßen für die Mitteilung seiner Einsichten an andere. Und wie eng beides zusammengehört, belegt ja schon der Rang der Gattung des Dialogs für Platons philosophische Schriften, in dem Gespräch und Selbstreflexion bis zur Ununterscheidbarkeit ineinander verwoben sind. ${ }^{17}$ Insofern besteht der sog. linguistic turn auch in

\footnotetext{
17 Ein besonders prägnanter Beleg für die Annahme einer grundsätzlichen Vorgängigkeit des Logos gegenüber aller Sprache findet sich in der christlichen Aneignung wie Umdeutung des antiken Logos. Augustinus hat ihr im letzten Buch seines Werks De trinitate mit der Unterscheidung zwischen dem inneren und dem äußeren Wort nicht nur eine präzise begriffliche Gestalt gegeben, er hat die Relation zwischen beiden Erscheinungsformen des Wortes zudem theologisch ausspekuliert und macht gerade dadurch den ontologischen Status des Logos der
} 


\section{einem Stück Selbstaufklärung der Philosophie. (Der Begriff der `Selbstaufklä- rung، ist womöglich dazu angetan Verwirrung zu stiften, könnte er doch den}

Sprache kenntlich. Die Sprachunabhängigkeit des Denkens ergibt sich für den Kirchenvater
schon allein aufgrund seiner Auffassung, daß das innere Wort nicht nur jeder Einzelsprache
voraufliege, sondern auch durch die Zuordnung wechselnder Lautkörper (oder anderer als
akustischer Zeichen) seine stets gleiche Identität bewahre. Einzig die Umstände einer Mittei-
lung scheinen dazu zu zwingen, dem inneren Wort einen materiellen Träger hinzuzufügen:
"Necesse est enim cum verum loquimur, id est quod scimus loquimur, ex ipsa scientia quam
memoria tenemus nascatur verbum quod eiusmodi sit omnino cuiusmodi est illa scientia de
qua nascitur. Formata quippe cogitatio ab ea re quam scimus verbum est quo in corde dicimus,
quod nec graecum est, nec latinum, nec linguae alicuius alterius; sed cum id opus est in
eorum quibus loquimur perferre notitiam, aliquod signum quo significetur assumitur. Et pler-
umque sonus, aliquando etiam nutus, ille auribus, ille oculis exhibetur, ut per signa corporalia
etiam corporis sensibus verbum quod mente gerimus innotescat. Nam et innuere quid est, nisi
quodam modo visibiliter dicere? (Augustinus: De trinitate, XV, 10, 19, hier zitiert nach: CCSL 50A, S. 486)« [»Wenn wir etwas Wahres sagen, d. h. etwas, das wir wissen, dann ist es notwendig, daß ein Wort aus dem Wissen, das wir im Gedächtnis festhalten, entsteht, das gänzlich von derselben Art ist wie das Wissen, aus dem es stammt. Das Denken der Sache, die wir wissen, ist nämlich das Wort, das wir im Herzen sprechen, das weder griechisch noch lateinisch ist und auch keiner anderen Sprache angehört. Aber weil es erforderlich ist, denen, zu denen wir sprechen, Kenntnis davon zu geben, greifen wir auf (irgend)ein Zeichen zurück, mit dessen Hilfe es bezeichnet wird. Zumeist ist dieses Zeichen ein Laut, mitunter auch eine Geste, ersterer richtet sich an die Ohren, letztere an die Augen, damit durch körperliche Zeichen auch den körperlichen Sinnen kundgetan werde, was wir in unserem Geist tragen. Denn was anderes ist es denn, eine Geste auszuführen, als in sichtbarer Weise etwas zu sagen?«] Augustins luzide Erkenntnis, daß die Sprache nicht des Lautes bedarf, sondern sich ebenso optischer Zeichen bedienen kann, markiert die Arbitrarität der signifiants, die für ihn freilich eine ganz andere Ursache als im Cours de linguistique générale besitzt. Bei Saussure resultiert die Arbitrarität des sprachlichen Zeichens aus der bloßen Funktionalität des Zusammenhangs von Signifikant und Signifikat, deren Begrifflichkeit allein schon ihre Interdependenz markiert. Für Augustinus ist die Arbitrarität der signifiants - eine doppelte Arbitrarität, die sich sowohl auf die einzelsprachliche Unterschiedlichkeit der Laute wie die mediale Variabilität des materiellen Zeichenträgers bezieht - hingegen eine Konsequenz der metaphysischen Stabilität des inneren Wortes, das allein um der Umstände einer Kommunikation willen nach einem körperlichen Ausdruck verlangt. Nur ein solchermaßen ontologisch stabiles inneres Wort aber erlaubt es, seine Beziehung zum körperlichen Wort der materiellen Kommunikation in Kategorien der Inkarnation zu denken: »Proinde verbum quod foris sonat, signum est verbi quod intus lucet, cui magis verbi competit nomen. nam illud quod profertur carnis ore, vox verbi est: verbumque et ipsum dicitur, propter illud a quo ut foris appareret assumptum est. Ita enim verbum nostrum vox quodam modo corporis fit, assumendo eam in qua manifestetur sensibus hominum; sicut Verbum Dei caro factum est, assumendo eam in qua et ipsum manifestaretur sensibus hominum. Et sicut verbum nostrum fit vox, nec mutatur in vocem: ita Verbum Dei caro quidem factum est, sed absit ut mutaretur in carnem. Assumendo quippe illam, non in eam se consumendo, et hoc nostrum vox fit, et illud caro factum est « (Augustinus: De trinitate, $\mathrm{XV}, 11.20$; CCSL 50A, S. 486f.). [»Deshalb ist das Wort, das außen erklingt, ein Zeichen des Wortes, 
Eindruck erwecken, als sei die im Zuge der Postulate des linguistic turns gewachsene metaphysische Skepsis ontologisch wahrer als die überkommene Metaphysik. Doch dem ist nicht so. Die Einsicht in einen intrinsischen Zusammenhang von Sprache und Denken sagt über die ontologische Qualität des Denkens nichts aus. Die Überzeugung von der Verwurzelung des Logos in der Welt unabhängig von unserem Denken wie die Annahme seiner bloßen - medial generierten Selbstbezüglichkeit sind ontologisch gleichermaßen wahrscheinlich resp. unwahrscheinlich.) Die mit dem linguistic turn verbundene Palastrevolution findet deshalb erst statt, als die überkommene Metaphysik radikale Veränderungen erfahren hat. Erst das auf sich selbst verwiesene Bewußtsein, das spätestens mit Kants Erkenntnistheorie sein ontologisches Fundament eingebüßt hat und in seiner Selbstbezüglichkeit gleichsam ortlos geworden ist, besinnt sich auf das Medium der Sprache als seinen angestammten Partner. Doch die unter diesen Voraussetzungen stattfindende Erneuerung des Bündnisses von Sprache und Denken ${ }^{18}$ kehrt die angestammte Beziehung zwischen ihnen um; und eben diese Inversion hat den sog. linguistic turn in die Welt gebracht. ${ }^{19}$

das drinnen leuchtet, dem die Bezeichnung als Wort weit mehr zukommt. Denn das, was mit dem fleischlichen Mund ausgesprochen wird, ist die Stimme des Wortes. Ein Wort wird auch dieses nur wegen des Wortes genannt, von dem es genommen ist, damit es draußen in Erscheinung treten kann. Solchermaßen nämlich wird unser Wort in gewisser Weise zur Stimme des Körpers, indem es die Gestalt annimmt, in der es den Sinnen der Menschen kundgetan werden kann. So ist auch das Wort Gottes Fleisch geworden, das er annahm, damit es darin sich selbst den Sinnen der Menschen offenbare. >Und wie unser Wort zur Stimme wird, ohne sich in Stimme zu verwandeln, so ist auch das Wort Gottes Fleisch geworden, doch keineswegs hat es sich in Fleisch verwandelt. Denn als es das Fleisch annahm, ist es darin nicht aufgegangen; und in diesem Sinn wird unser Wort zur Stimme wie das göttliche zu Fleisch geworden ist.«] Vielleicht findet die Differenz zwischen dem kontingenten materiellen Zeichen und dem inneren Wort seinen sinnfälligsten Ausdruck im Gegensatz der beiden Verben, mit denen ihre jeweilige Erscheinungsweise hier gekennzeichnet wird: Das äußerliche Wort klingt (sonat), das innere leuchtet (lucet): Es strahlt in jenem Licht, das aller Körperlichkeit entzogen bleibt und in dem auch Gott seine ewige Wohnstatt hat.

18 Die progredierende Mathematisierung der Naturerkenntnis ist nur eines der Symptome der Aufkündigung des überkommen Bündnisses von Sprache und Denken bei und seit Descartes. Kants Einsicht, daß die Erkenntnis urteilsförmig ist, befördert gerade seine ontologischen Reserven.

19 Ich benutze den Begriff in diesem Artikel in einem weiteren Sinn, als ihn Richard Rorty verwendet, durch den er bekanntlich - im Anschluß an Gustav Bergmann - zu einem terminus technicus der Charakteristik einer der wesentlichen Veränderungen der Philosophie im 20. Jahrhundert geworden ist (vgl. R. M. Rorty: Metaphilosophical Difficulties of Linguistic Philosophy. In: The Linguistic Turn. Essays in Philosophical Method, edited by Richard M. Rorty. Chicago University Press 1992, S. 1-39). Im engeren Sinn seines Begriffsgebrauchs ist darunter die Wendung zu einer Konzeption von Philosophie verstanden, die auf der An- 
Sie zieht zugleich höchst riskante Folgen für das Denken nach sich. Denn die Sprache, die sozusagen vom Status einer Hilfskraft des Denkens in die Position des Lehrstuhlinhabers wechselt, vermag dem Bewußtsein letztlich keine Stütze, sondern nur ein Instrument seiner Selbstartikulation an die Hand zu geben. Aus diesem Grund mündet die Reflexion über das Verhältnis des Denkens zur Sprache im Zeichen des linguistic turns weithin in eine Erläuterung seiner eigenen Grundlosigkeit. $\mathrm{Zu}$ deren Erkundung wie Bezeichnung hat das Sprachdenken des 20. Jahrhunderts eine Reihe von sehr prägnanten - und nicht zuletzt darum wohl prominent gewordenen - Formeln entwickelt. Sie reichen von Wittgensteins Sprachspiel bis zu Derridas Neologismus der différance mit ihrer unorthodoxen Graphie eines $a$ in der Wortendung.

Kennzeichnend für diese Formeln ist es, daß sie die Analyse der Sprache und ihrer Mechanismen mit einer Demonstration ihrer mangelnden metaphysischen Deckung verbinden. Beides scheint in der Perspektive der mit ihnen verbundenen Sprachkonzeptionen zusammenzugehören, ja die beiden Seiten derselben Sache zu bilden. In der Konsequenz der Verbindung beider theoretischer Anliegen aber gerät die Sprache letztlich selbst zu einem Organ der Demonstration ihrer ontologischen Grundlosigkeit.

Bei aller Unterschiedlichkeit der Konzeptionen, denen die Begriffe Sprachspiel und différance ${ }^{20}$ jeweils entstammen, weisen sie einige bemerkenswerte

nahme beruht, ihre überkommenen Problemstellungen als Probleme der Sprache beschreiben zu können. Der Ausdruck linguistic turn bleibt in dieser Bedeutung also vor allem auf eine bestimmte Form von Sprachphilosophie bezogen. Ich verstehe darunter hingegen eine allgemeine Tendenz im Denken des 20. Jahrhunderts, (zunächst) die Sprache (und in ihrem Gefolge weitere Medien) als maßgebliche Determinante der Wirklichkeitserkenntnis zu begreifen. Der linguistic turn erweist sich insofern als Einstieg in eine Bewegung des Denkens, die jegliche Rationalität als medienabhängig denkt. Sie erfaßt in diesem Sinn auch weitere Bereiche der Philosophie als die Sprachphilosophie. So lassen sich in Heideggers Fundamentalontologie durchaus Elemente einer solchen >medialen etwa bei seiner Charakteristik des Daseins als eines Verstehens, das auf Auslegung gründet: Die Explikation eines Kernkonzepts von Heideggers Denken, des Daseins, erfolgt mithin im Rückgriff auf eine textuelle Praxis (vgl. hierzu des Näheren meinen in Anm. 7 zitierten Artikel). Das hier skizzierte allgemeinere Verständnis des linguistic turns lädt auch dazu ein, die Konsequenzen seiner Rückwirkung auf das Konzept der Sprache selbst intensiver zu reflektieren.

20 Ich verwende auch im Deutschen die französische Form des Begriffs, da ich mich zum orthographischen Barbarismus der »Differänz«, die mancherorts üblich geworden ist, nicht bereitfinden kann - und dies umso weniger, als Substantive mit der Endung -ance im Französischen völlig geläufig sind, im Deutschen hingegen nicht. Da müßte kann schon auf dialektale Vorbilder wie die »Pänz« zurückgreifen - eine im Rheinland übliche Bezeichnung für Kinder. (Der Ausdruck kann sogar auf eine lateinische Etymologie [pantex] zurückblicken, 
Gemeinsamkeiten auf. Dies betrifft zunächst eine strukturelle Analogie zwischen ihnen. Wittgenstein wie Derrida unterlaufen eine kategoriale Opposition, die andernorts für die Reflexion über die Sprache im 20. Jahrhundert eine enorme Bedeutung gewonnen hat: Saussures Unterscheidung zwischen langue und parole. Derrida tut dies sehr bewußt in Kenntnis von und im Rahmen einer ausdrücklichen Auseinandersetzung mit dem Cours de linguistique générale. Bei Wittgenstein bleibt es bei einer konzeptuellen Korrespondenz mit Saussures Kategorien, deren Zuordnung in den Philosophischen Untersuchungen indessen gerade anders als im Cours angelegt ist. Schon die erste Verwendung des Begriffs Sprachspiel in Wittgensteins Schrift vermag diesen Unterschied plastisch zu belegen:

7. In der Praxis des Gebrauchs der Sprache (2) ruft der eine Teil die Wörter, der andere handelt nach ihnen; im Unterricht der Sprache aber wird sich dieser Vorgang finden: Der Lernende benennt die Gegenstände. D. h. er spricht das Wort, wenn der Lehrer auf den Stein zeigt. - Ja, es wird sich hier die noch einfachere Übung finden: der Schüler spricht die Worte nach, die der Lehrer ihm vorsagt - beides sprachähnliche Vorgänge.

Wir können uns auch denken, daß der ganze Vorgang des Gebrauchs der Worte in (2) eines jener Spiele ist, mittels welcher Kinder ihre Muttersprache erlernen. Ich will diese Spiele »Sprachspiele« nennen, und von einer primitiven Sprache manchmal als einem Sprachspiel reden.

Und man könnte die Vorgänge des Benennens der Steine und des Nachsprechens des vorgesagten Wortes auch Sprachspiele nennen. Denke an manchen Gebrauch, der von Worten in Reigenspielen gemacht wird.

Ich werde auch das Ganze: der Sprache und der Tätigkeiten, mit denen sie verwoben ist, das »Sprachspiel« nennen. ${ }^{21}$

Man wird über die Konsequenzen der Vorentscheidungen gewiß streiten können, die bereits mit Wittgensteins Ausgangshypothese, mit seinem Postulat einer weitgehenden (wo nicht grundsätzlichen) Analogie (wo nicht Identität) von Spracherwerb und Sprachgebrauch, für sein Konzept von Sprache getroffen werden. Aber wie immer es um die Angemessenheit dieser Prämissen bestellt sein mag, der zitierte Paragraph aus den Philosophischen Untersuchungen ist durch eine beständige Erweiterung des Begriffs des Sprachspiels gekennzeichnet.

deren Semantik ihn allerdings kaum adelt.) Zu Derridas Begriff der différance vgl. Kablitz: Vom Nutzen und Nachteil eines Neologismus: Derridas différance. In: Sprache und Literatur 49 (2020), Heft 122, S. 297-332.

21 Ludwig Wittgenstein: Philosophische Untersuchungen. Hier zitiert nach: Ders.: Tractatus logico philosophicus. Tagebücher 1914-1916. Philosophische Untersuchungen (Werkausgabe, Band I). Frankfurt a.M. 2006, hier S. 241. 
Genauer betrachtet, beruht die Bestimmung dieses Begriffs auf einer gegenläufigen Bewegung von Eingrenzung und Erweiterung. Denn zunächst nimmt sich das Sprachspiel, mit dessen Hilfe Kinder eine Sprache erlernen, wie eine Variante jener Spiele aus, die für ihr Lebensalter schlechthin charakteristisch sind. ${ }^{22}$ Diese erste Zuordnung des Sprachspiel-Begriffs mit seiner doppelten Lesbarkeit ist im Übrigen alles andere als nebensächlich. Sie sorgt vielmehr dafür, daß er keine metaphorische Basis hat, sondern als ein Spiel im letztlich anthropologisch begründeten - wörtlichen Sinn zu verstehen ist.

Das solchermaßen eingeführte Sprachspiel aber wird sodann konsequent mit neuen Anwendungsmöglichkeiten seiner Bezeichnung versehen: Von »einer primitiven Sprache« möchte Wittgenstein »manchmal als einem Sprachspiel reden«. Sodann wird der Begriff auf bestimmte Praktiken des Sprechens ausgeweitet, bis im Schlußsatz »das Ganze der Sprache und der Tätigkeiten, mit denen sie verwoben ist « zum Sprachspiel erklärt wird. Dieses Ganze aber weist eine deutliche Nähe zu Saussures Begriff der langue auf, der die Tätigkeiten der parole gegenübergestellt sind. Sprachsystem und Sprachgebrauch aber gehen solchermaßen - und darin liegt der Unterschied gegenüber dem Cours de linguistique générale - in demselben Begriff eine Synthese ein, in der sie schwer auseinanderzuhalten sind.

Wenn ich hier von einem System im Blick auf das Spiel spreche, dann scheint dies insofern berechtigt, als ja auch jedes Spiel Regeln unterliegt - Regeln, die jeder Spieler zu beachten hat, wenn er es spielt. Aber aus diesem Zusammenhang die Schlußfolgerung zu ziehen, daß das Spiel und das Spielen von gleicher Art sind - und eben dies suggeriert ja Wittgensteins auf beides zu applizierender Begriff des Sprachspiels - bleibt eine riskante Conclusio. Denn Interdependenz bedeutet etwas anderes als (semantische) Identität. Daß die Praxis des Spielens (wie der Sprache) eine Veränderung ihrer Regeln zur Folge haben kann (und hat), steht außer Frage, wie ebenso kaum zu bestreiten sein wird, daß die Spielregeln das Spielen eines Spiels maßgeblich bestimmen und Abweichungen nur innerhalb gewisser Grenzen zulassen. Doch eben diese Interdependenz setzt auch die sachliche (wie konzeptuelle) Differenz zwischen dem, was sich in wechselseitiger Abhängigkeit befindet und aus genau diesem

22 In diesem Sinne scheint mir von Belang zu sein, daß die Wendung »eines jener Spiele« sich im Grunde in doppelter Weise - gleichsam in einem analytischen wie in einem synthetischen Verständnis - lesen läßt. Sie kann besagen: ১Jene Spiele, von denen wir ja wissen, daß Kinder mit ihrer Hilfe die Sprache erlernen`. Sie kann sich aber ebenso auf `diejenigen unter den Spielen der Kinder, mit denen sie die Sprache erlernen beziehen und damit diese Sprachspiele unter das allgemeine kindliche Spielverhalten subsumieren. Die metonymische Nähe von Kind und Spiel jedenfalls ist auffällig. 
Grund die Möglichkeit besitzt, einander $\mathrm{zu}$ beeinflussen, voraus. Gerade sie aber wird durch eine Subsumtion von beidem unter denselben Begriff nivelliert (wo nicht negiert).

Indessen besitzt der Begriff des Spiels neben seiner Implikation einer bestimmten Regelhaftigkeit eine weitere semantische Facette. Sie beruht auf dem Gegensatz zwischen Ernst und Spiel: ‘Es ist ja nur ein Spiel!.${ }^{23}$ Wird auch diese Dimension seiner Bedeutung bei Wittgenstein relevant?

Die Antwort auf diese Frage fällt im Blick auf die Philosophischen Untersuchungen nicht leicht, da ein solcher Gegensatz für den Sprachspielbegriff nicht ausdrücklich hergestellt wird. Indessen gehört dessen Verwendung bei Wittgenstein zur Charakteristik der Sprache zugleich zu seinem Feldzug gegen ein Konzept der Sprache, das dem Begriff der Bedeutung mehr als nur das semantische Potential eines Wortgebrauchs zubilligen möchte. Spiel meint in diesem Sinn gleichsam emphatisch den bloßen Gebrauch, dem jede Vorgängigkeit irgendeiner Entität, die ihn determinierte, fehlt - dem vor allem jede metaphysische Stabilität fremd ist. ${ }^{24}$ In diesem Sinn gehört auch das bloße Spiel, dem der Ernst (der - ontologisch verstandenen - Wirklichkeit) nachgerade programmatisch fehlt (und zu fehlen hat) zum Bedeutungsspektrum von Wittgensteins Sprachspiel. Es ist auch ein Remedium gegen alle Ontologie. Insofern gehören in den Philosophischen Untersuchungen die Analyse der Mechanismen der Sprache und die Zurückweisung aller metaphysischen Ansprüche für sie zueinander.

Wittgensteins antimetaphysische Kampagne zielt bekanntermaßen nicht zuletzt auf seine eigene Vergangenheit:

114. Logisch-Philosophische Abhandlung 4.5 »Die allgemeine Form des Satzes ist: Es verhält sich so und so«. - Das ist ein Satz von jener Art, die man sich unzählige Male wiederholt.

23 Es scheint, als besitze der Spielbegriff diese Implikation der Regelhaftigkeit nicht grundsätzlich, sondern dort, wo man ihn zum Objekt des Verbs spielen machen kann. Wird er absolut gesetzt (`Die Kinder spielen`), gilt dies hingegen nicht. Dort markiert er den Gegensatz zum Ernst.

24 »116. Wenn die Philosophen ein Wort gebrauchen - »Wissen«, »Sein«, »Gegenstand«, "Ich «, "Satz«, »Name« - und das Wesen des Dings zu erfassen trachten, muß man sich immer fragen: Wird denn dieses Wort in der Sprache, in der es seine Heimat hat, je tatsächlich so gebraucht? - Wir führen die Wörter von ihrer metaphysischen, wieder auf ihre alltägliche Verwendung zurück« (Wittgenstein: Philosophische Untersuchungen (s. Anm. 21), S. 300). Wer auch immer sich hinter dem (betonten) Wir - einer gleichsam nietzscheanischen Geste der Abgrenzung gegenüber den Philosophen - verbergen mag, er spielt den (Sprach-)Gebrauch gegen jedwede Ontologie aus (oder soll es zumindest tun, sofern das Personalpronomen keinen Pluralis Majestatis bedeutet, sondern eine Gruppe von Einvernehmlichen bezeichnet - oder beschwört). 
Man glaubt, wieder und wieder der Natur nachzufahren, und fährt nur der Form entlang, durch die wir sie betrachten. ${ }^{25}$

Gegen den Verfasser des Tractatus logico-philosophicus aber hatte der Autor der Philosophischen Untersuchungen schon zuvor Klage geführt. ${ }^{26}$ Und bereits im Vorwort heißt es:

Vor zwei Jahren aber hatte ich Veranlassung, mein erstes Buch (die »Logisch-Philosophische Abhandlung") wieder zu lesen und seine Gedanken zu erklären. Da schien es mir plötzlich, daß ich jene alten Gedanken und die neuen zusammen veröffentlichen sollte: daß diese nur durch den Gegensatz und auf dem Hintergrund meiner älteren Denkweise ihre rechte Beleuchtung erhalten könne. Seit ich nämlich vor 16 Jahren mich wieder mit Philosophie zu beschäftigen begann, mußte ich schwere Irrtümer in dem erkennen, was ich in jenem ersten Buch niedergelegt hatte. ${ }^{27}$

Es ist hier nicht der Ort im Einzelnen darüber nachzudenken, inwieweit die Philosophischen Untersuchungen als Palinodie des Tractatus diesem Text zumal in ihrem dezidiert antimetaphysischen Gestus e negativo noch immer verhaftet bleiben. Eine Konsequenz dieses nicht zuletzt dadurch motivierten Feldzugs gegen alle Ontologie könnte sich allerdings in einer Blindstelle der mit ihm verbundenen Strategie, präziser gesagt: in einem performativen Widerspruch äußern, in den er mündet. Denn, wenn gilt, daß Sprache schlechthin aus Sprachspielen besteht, mit welchem Recht läßt sich unter diesen Bedingungen das Sprachspiel der Metaphysik als ein minderwertiges begründen, ja ihm womöglich seine Berechtigung absprechen?

Als Maßstab der Akzeptabilität eines Wortes führt Wittgenstein selbst die Frage an: »Wird denn dieses Wort in der Sprache, in der es seine Heimat hat, je tatsächlich so gebraucht? « Beachtung verdient vor allem das Adverb »je«. Die Bedingungen dafür, daß ein Sprachspiel als ein solches gelten kann, sind auffällig niedrig gehalten. Im vorliegenden Fall muß man die betreffende Frage also sehr entschieden mit $J a$ beantworten. Denn »die Philosophen «, zumindest etliche unter ihnen, haben die zitierten Wörter »tatsächlich so gebraucht«. Den Philosophischen Untersuchungen liegt eine antimetaphysische Inbrunst zugrunde, für deren Befriedigung das in dieser Schrift beschriebene Sprachkonzept nicht nur keine Voraussetzungen bereitstellt, es stellt sie vielmehr gerade in Frage.

Wenn das Sprachspiel als Waffe gegen den metaphysischen Ernst wirken soll, so stellt es doch ein ausgesprochen zweischneidiges Schwert dar. Sein relati-

25 Wittgenstein: Philosophische Untersuchungen (s. Anm. 21), S. 300.

26 Vgl. § 23.

27 Wittgenstein: Philosophische Untersuchungen (s. Anm. 21), S. 232. 
vierender Universalismus macht nämlich insgeheim wieder möglich, was er gerade verhindern soll. Die Metaphysik ist - aus Sicht dieses Begriffs betrachtet - eben auch ein Sprachspiel - eines unter anderen. Und weil dem so ist, ist es nicht weniger wahr - freilich auch nicht wahrer - als andere Sprachspiele, sondern - eben ein Spiel. Und dieser Befund gilt selbstredend auch für das Sprachspiel der Philosophischen Untersuchungen. Sogar die Beschwörung des Sprachgebrauchs als Instanz einer Überlegenheit alltäglicher gegenüber metaphysischer Rede verliert in der Konsequenz dieser Gegebenheiten ihre Grundlage. Vor allem dann, wenn auch das »Ganze der Sprache« ein Sprachspiel ist. Die Frage nach der Wahrheit stellt sich im Grunde gar nicht mehr. Jedenfalls nicht mehr für ein Sprachspiel. Sie stellt sich allenfalls noch innerhalb eines Sprachspiels.

Wenn auch Derridas Begriff der différance die Opposition von langue und parole aufhebt, dann tut er dies jedoch im gegenteiligen Sinn dessen, was sich in den Philosophischen Untersuchungen beobachten läßt: In seinem Fall wird nicht das Regelsystem der Sprache auf den Sprachgebrauch reduziert, vielmehr wird umgekehrt die parole zum ausführenden Organ der langue erklärt. Es ist dies eine Konsequenz der Relation des Neologismus différance zu dem von Saussure übernommenen Begriff der différence, den Derrida mit seiner Wortprägung nicht nur zu transformieren, sondern letztlich zu ersetzen anstrebt.

Die différence besitzt bekanntlich eine wesentliche Rolle für Saussures Konzept des Sprachsystems, stellt sie doch nichts weniger als das konstitutive Merkmal der langue schlechthin dar: »[D]ans la langue il n'y a que des différences ${ }^{28}{ }^{28}$ Ein wesentliches Anliegen von Derridas Sprachtheorie besteht darin, diesem Konzept der différence die Stabilität und damit zugleich seine Konsistenz streitig zu machen. Dies aber geht nicht ohne eine gehörige Bearbeitung

28 Ferdinand de Saussure: Cours de linguistique générale. Édition critique préparée par Tullio de Mauro. Paris 1974, S. 166. [»In der Sprache gibt es nur Unterschiede.«] Die Zuordnung des Autors Ferdinand de Saussure zum Cours de linguistique générale läßt sich allerdings im Lichte der jüngeren Forschung nicht mehr so einfach vornehmen, wie dies lange Zeit üblich war. Seit jeher ist bekannt, daß es sich bei diesem Band nicht um eine von Saussure selbst verfaßte Monographie handelt, sondern um ein auf der Grundlage von Vorlesungsmitschriften von seinen Schülern Charles Bally und Albert Sechehaye zusammengestelltes Buch. Sie selbst haben diese Vorlesungen allerdings gar nicht besucht. Vor allem den Forschungen von Ludwig Jäger ist es zu danken, daß inzwischen erhebliche Diskrepanzen zwischen der nachweislich von Saussure stammenden und vornehmlich aus seinen Notes zu rekonstruierenden Theorie der Sprache und der im Cours nachzulesenden Sprachtheorie bemerkt worden sind. Vgl. hierzu die grundlegenden Untersuchungen L. J.: F. de Saussures historisch-hermeneutische Idee der Sprache. Ein Plädoyer für die Rekonstruktion des saussureschen Denkens in seiner authentischen Gestalt. In: Linguistik und Didaktik 27 (1976), S. 210-244 sowie ders.: Ferdinand de Saussure. Zur 
von Saussures Begriff vonstatten, den Derrida recht entschieden für seine Zwecke präpariert:

On en tirera cette première conséquence que le concept signifié n'est jamais présent en lui-même, dans une présence suffisante qui ne renverrait qu'à elle-même. Tout concept est en droit et essentiellement inscrit dans une chaîne ou dans un système à l'intérieur duquel il renvoie à l'autre, aux autres concepts, par jeu systématique de différences. Un tel jeu, la différance, n'est plus alors simplement un concept mais la possibilité de la conceptualité, du procès et du système conceptuels en général. ${ }^{29}$

Mit dem Auftritt der différance in den zitierten Worten ist Derrida augenscheinlich dort angelangt, wohin es bei seiner Charakteristik der saussureschen différence gehen soll. Auf welchem Weg aber kommt er dorthin?

Einen entscheidenden Schritt in Richtung auf dieses Ziel bietet die Übersetzung von Saussures Konzept des signifié in Derridas eigene Begrifflichkeit der Präsenz. In diesem Sinn heißt es dazu bei ihm: »[D]aß das bezeichnete Konzept niemals in sich selbst anwesend ist « (que le concept signifié n'est jamais présent en lui-même). Diese Charakteristik des signifié geht zugleich mit einer nicht unerheblichen Reinterpretation der différence einher, heißt es doch von ihr, sie befinde sich nicht »in einer hinreichenden Anwesenheit, die nur auf sich selbst verwiese« (dans une présence suffisante qui ne renverrait qu'à elle-même). Auch wenn sich die betreffende Reinterpretation sozusagen sunter der Hand vollzieht und gleichsam im Schutzraum einer als solcher vorausgesetzten Selbstverständlichkeit stattfindet, die konzeptuelle Verschiebung, die sich vollzieht, ist folgenreich. Denn die der Differenz zugrundeliegende logische Relation einer Opposition wird von Derrida ohne Umschweife als eine zeichenhafte Beziehung gedeutet, präziser gesagt: in eine semiotische Relation umgedeutet.

Doch diese Gleichsetzung der beiden Typen von Relationen entbehrt einer jeglichen sachlichen Grundlage. Logische Beziehungen gründen auf Verhältnissen

Einführung. Hamburg 2010. Ohne in diesem Zusammenhang auf die von Jäger aufgezeigten Unterscheidungen im Einzelnen eingehen zu können, werde ich deshalb vom Cours de linguistique générale (meist abgekürzt als Cours) als einem Werk Saussures nur unter jenem Vorbehalt sprechen, den Jägers Untersuchungen erforderlich gemacht haben, und seine Bezeichnung als ein Buch von Saussure weitgehend vermeiden.

29 Jacques Derrida: La différance. In: ders.: Marges de la philosophie. Paris 1972, S. 1-29, hier S. 11. [»Man wird daraus als erste Konsequenz ableiten, daß das bezeichnete Konzept niemals in sich selbst anwesend ist, in einer hinreichenden Anwesenheit, die nur auf sich selbst verwiese. Jedes Konzept ist in der Tat und seinem Wesen entsprechend einer Kette eingeschrieben oder einem System, innerhalb dessen es auf das andere, auf die anderen Konzepte durch ein systematisches Spiel von Unterschieden verweist. Ein solches Spiel, die différance, ist deshalb nicht einfach ein Konzept, sondern die Möglichkeit eines Konzeptes, des Prozesses und Systems des Konzepts im Allgemeinen.«]. 
der Implikation, die von Zeichenbeziehungen grundsätzlich zu unterscheiden sind: Die Glieder einer Opposition verweisen nicht aufeinander, der sie konstituierende Unterschied zwischen ihren Gliedern wird vielmehr vorausgesetzt.

Nur um den Preis dieser prekären Identifikation von Logik und Semiotik scheint es Derrida jedoch zu gelingen, die différence Saussures mit seiner différance $\mathrm{zu}$ amalgamieren, ja letztere als die Grundlage aller différences zu postulieren. Denn erst unter dieser Voraussetzung erscheint es möglich, die einzelnen Terme einer Opposition als mit sich selbst nicht identische Größen auszuweisen, sie deshalb in eine unendliche Kette von Verweisen hineinzuholen und damit semantischer Dispersion den Weg zu bahnen. Erst auf diese Weise kommt auch die Zeitstruktur eines Aufschubs zustande, der die différance von der différence unterscheidet und die Auflösung aller semantischen Stabilität betreibt. Auch an dieser Verzeitlichung des signifié ist noch einmal die semiotische Reinterpretation der logischen Oppositionsrelation beteiligt. Denn ein implikatives Verhältnis ist gleichsam zeitlos. Ein wechselseitiger Verweis aufeinander bildet hingegen einen unabschließbaren Prozeß, auf dessen Grundlage sich dann die unaufhaltsame Verschiebung der Semantik eines Lexems vollziehen kann.

Es lohnt, etwas genauer darüber nachzudenken, was différance, in Sinne eines Aufschubs, an dieser Stelle genau besagen will. Was nämlich wird im Falle des signifié eigentlich verschoben? Folgt man Derridas Argumentation konsequent, dann wäre dies im Grunde doch wohl etwas, das es nie gegeben hat, nämlich eine mit sich selbst identische Bedeutung. Der Aufschub von etwas nie Existentem aber kommt im Grunde einer Suche gleich. Im Blick auf das signifié nimmt die différance folglich die Gestalt einer quête an; denn der Aufschub von etwas Unbekanntem kann letztlich nur eine Suche nach seiner Bestimmung als solcher sein. Dadurch aber gerät der Sprachgebrauch zu einem Annex der Theorie, als hätte er seinen heimlichen Antrieb in einer Leerstelle des Systems (sofern von einem solchen überhaupt noch die Rede sein kann) als stellte er sich der (freilich stets schon zum Scheitern verurteilten) Aufgabe, das einzuholen, was sich nicht einholen läßt.

Der Aufschub, für den der Neologismus der différance im Wortsinn steht, verwandelt sich insofern konsequent in semantische Dispersion: in eine Zerstreuung aller Bedeutung, die umso erfolgreicher ihre, wenn auch aussichtslose, Suche zum Erfolg führen zu können scheint, je breiter sie angelegt ist - je größer also die Variation der Bedeutung(en) ausfällt. Denn Erfolg wird sie gerade haben, wenn ihr derselbe im Sinn eines erreichbaren Endziels versagt bleibt. Aufschub verwandelt sich folglich in einen Prozeß bloßer Dispersion. Aber vermag ein solches Konzept, das den Sprachgebrauch gleichsam als eine Summe von Ausfüh- 
rungsbestimmungen zu den Verwerfungen des Systems begreifen läßt, wirklich den Erfordernissen von Sprechsituationen und ihren jeweiligen Informationsbedürfnissen gerecht $\mathrm{zu}$ werden?

Hier bestätigt sich, was wir eingangs unseres Blicks auf Derrida postuliert hatten: Wenn Derrida - in umgekehrter Richtung wie Wittgenstein - die Opposition zwischen langue und parole aufhebt, dann ebnet er sie nicht von der Seite des Sprachgebrauchs herkommend ein, vielmehr wird die parole gleichsam zu einer Agentin der Verwerfungen einer langue, die um ihrer mangelnden Konsistenz willen den Anspruch auf einen solchen Begriff letztlich verliert. Deutlicher noch als in Wittgensteins Philosophischen Untersuchungen läßt ein Konzept der Sprache im Zeichen der différance ihre Mechanismen mit dem ihr zugedachten antimetaphysischen Impetus zusammenfallen. Diesmal verschwindet das System der langue nicht in einer parole, die im Grunde nichts anderes mehr zur Grundlage hat als die schlechthin unabsehbare Empirie ihres Gebrauchs und deshalb alle Regeln der Sprachspiele auf ein bloßes Postulat ihrer Existenz verschieben muß (ohne sie doch beschreiben zu können als stünde eine beschreibbare Regelhaftigkeit im Ruch einer ontologieverdächtigen Stabilität, einer Entität, die doch dem Gebrauch vorausläge). Bei Derrida steht Wittgensteins Abdikation der Theorie vor dem Sprachgebrauch hingegen die Vereinnahmung der parole für ein Anliegen der Theorie gegenüber, zu deren Ausführungsorgan sie gerät.

Die literaturwissenschaftlich zentrierte Kulturwissenschaft setzt sehr konsequent die beiden skizzierten Konsequenzen des linguistic turns um, der ebenso ein Ermächtigungsgesetz mit sich führt, wie er zu ontologischer Demission nötigt. Beides aber gehört, wie wir sahen, zusammen. So scheint sich die Wissenschaft von der Literatur in der jüngeren Vergangenheit dazu ermächtigt zu fühlen, von letztlich jedwedem Gegenstand zu handeln. Und wenn denn gilt, oder gälte, daß die Sprache den Zugang zur Welt bestimmt, was scheint da näher zu liegen als die Annahme der universellen Zuständigkeit einer sprachbasierten Wissenschaft? Allerdings begibt sie sich auf diese Weise in eine gefährliche Nähe zu Ionescos doctorat total. (Streng genommen gehört zu einer solchermaßen verstandenen Kulturwissenschaft auch der Anspruch auf die Objekte der Naturwissenschaft, denn schließlich ist auch der Zugang zu ihnen kulturell geprägt.)

Aber nicht nur die Entgrenzung des überkommenen Fundus philologischer Gegenstände läßt sich als eine Konsequenz des linguistic turns begreifen. Gleiches gilt für die ebenso unverkennbare Tendenz zu einer Entsubstantialisierung, in der sich das Postulat ontologischer Grundlosigkeit spiegelt. Vielleicht kommt diese Tendenz nirgends deutlicher als in den Gender Studies zum Ausdruck, die sich ja zum Kernbereich der Cultural Studies zählen dürfen. 
Ihre Stoßrichtung ist wohl bekannt. $\mathrm{Zu}$ ihren zentralen Anliegen gehört der Nachweis, daß das biologische Geschlecht zu weiten Teilen ein kulturelles und deshalb auch zur Disposition stehendes - Konstrukt darstellt, mithin nur vermeintlich natürlich begründet sei. ${ }^{30}$ Womöglich bieten deshalb die Gender Studies ein besonders geeignetes Exempel, um $\mathrm{zu}$ studieren, wie sich der sprachbasierte ontologische Skeptizismus zu einem generellen methodischen Prinzip wandelt und ausweitet. Denn in und mit den in ihrem Rahmen betriebenen Forschungen wird der sprachliche Umgang mit der Welt auf den praktischen Umgang mit ihr ausgedehnt. In ihrem Fall steht weit mehr als die bloße Rede über die Wirklichkeit zur Diskussion wie zur Disposition. Auch das (überkommene) Handeln ist in Frage gestellt. Es scheint der Mühe wert zu sein, sowohl die Schlüssigkeit wie die Risiken dieses Anspruchs genauer zu prüfen.

Beginnen wir mit ersterem. Wenn denn gilt, daß die Sprache allen Zugang zur Wirklichkeit bestimmt, ist auch die Geschlechterdifferenzierung, so lautet die entsprechende Schlußfolgerung, sprachlichen Ursprungs. Aber diese Conclusio zielt über ihr Ziel hinaus. Denn sie verwandelt skeptischen Gewißheitsverzicht in rebellische Gewißtheit. Alles, was sich zulässigerweise sagen läßt, beschränkt sich auf die Feststellung, daß es ungewiß ist, ob die Unterscheidung der Geschlechter eine andere als sprachliche - und damit kulturelle - Grundlage hat. Und dies aus einem naheliegenden Grund: Denn auch die Überprüfung der These vom grundsätzlichen Konstruktcharakter des Geschlechts ist ihrerseits von der Sprache abhängig. Hier lauert also ein circulus vitiosus. Auch die kulturelle Vielfalt der Geschlechterrollen ist kein notwendiges Gegenargument gegen biologische Konditionierung. Denn was spricht dagegen, daß biologische Prämissen in kultureller unterschiedlicher Weise wirksam werden? Und so bleibt im Grunde kaum mehr als die Zuverlässigkeit der Skepsis, daß eine biologische Grundlage der gender-Differenzierung keinesfalls gewiß ist. Das Postulat der Konstruktionsgewißheit stellt hingegen eine von der Logik der Prämisse nicht gedeckte Schlußfolgerung dar. Es mündet im Grunde in einen Substantialismus, der sich gerade anti-substantialistisch versteht.

Nun ist der Bereich der Gender Studies für die Analyse der Kulturwissenschaft ein in mehrfacher Hinsicht höchst aufschlußreiches Terrain, und dies vor

30 Judith Butler hat die wesentlich normative diskursive Basis der kulturellen Produktion der Kategorie des Geschlechtes sehr deutlich benannt: „Sexual difference, however, is never simply a function of material differences which are not in some way both marked and formed by discursive practices. Further, to claim that sexual differences are indissociable from discursive demarcations is not the same as claiming that discourse causes sexual difference. The category of 'sex is, from the start, normative, it is what Foucault has called a >regulatory ideal« « (Judith Butler: Bodies that Matter. On the Discursive Limits of »Sex «. New York, London 1993, S. 1). 
allem deshalb, weil die These vom Konstruktcharakter des biologischen Geschlechtes zugleich an offenkundige Grenzen stößt. Denn es läßt sich schwerlich bestreiten, daß bestimmte biologische Funktionen an den Unterschied der Geschlechter gebunden sind. Dieser Umstand bietet deshalb zum einen so etwas wie eine konzeptuelle Herausforderung, verlangt er doch (und ermuntert zugleich dazu), die Grenzen des Konstruktivismus auszutesten und seinen zumindest partiellen Belang auch und gerade dort unter Beweis zu stellen, wo seine Plausibilität sich besonders heftigen Widerständen ausgesetzt findet.

Was sich hier vollzieht, ist augenscheinlich ein Kampf um Zuständigkeiten, der indessen weiteren Aufschluß über das zugrunde liegende Programm gestattet. Die für die Gender Studies charakteristische Überführung des theoretischen in ein praktisches Postulat, dessen Ansprüche auf logisch, wie gesehen, eher tönernen Füßen stehen, sofern sie sich auf die Gewißheit einer ausschließlich diskursiv-kulturellen Grundlage berufen, geben zu erkennen, daß dieses praktische Postulat keine bloße Resultante der Theorie ist, sondern die Theorie selbst ein Stück weit dem praktischen Ziel angepaßt wird. Gerade hier tritt jener Konnex von Episteme und Macht zutage, der bekanntlich niemand anderen intensiver als Michel Foucault beschäftigt hat. Augenscheinlich ist er auch der Kulturwissenschaft, ungeachtet ihres emanzipatorischen Anspruchs (ja vielleicht gerade um dessentwillen) keineswegs fremd.

Daß die Zumutungen des Patriarchats längst jegliche Berechtigung verloren haben, dürfte inzwischen in unserem Kulturkreis bei jedem, der nur halbwegs bei Verstand ist, angekommen sein. Ob indessen eine ein Stück weit erschlichene, zumindest aber in ihrer Geltung prekäre Theorie eine besonders glückliche Stütze für legitime Machtinteressen darstellt, steht dahin. Das Risiko, das in einer solchen Verquickung, wo nicht Permutation von Erkenntnis und Interesse steckt, besteht nicht zuletzt darin, daß die Defizite des theoretischen Anspruchs die Legitimität des sozialen Anspruchs nachhaltig beschädigen können.

Wenn ich Entgrenzung und Entsubstantialisierung als die beiden aus dem linguistic turn stammenden Grundtendenzen der Kulturwissenschaft beschrieben habe, dann ist freilich zu bedenken, daß sie nicht unabhängig voneinander sind, sondern unmittelbar miteinander zusammenhängen. Wir haben dies anhand der Sprachtheorie bemerken können. Und dieser Zusammenhang ist evident. Fehlt nämlich den Eigenschaften der Dinge eine substantielle Grundlage, so scheinen sie auf das Medium zu verweisen, dem die Bestimmung ihrer Merkmale entstammt. Ebenso aber gilt, daß alles, was die Sprache bezeichnet und bezeichenbar macht, auf nichts anderem als eben ihrer Zuschreibung beruht und damit letztlich im Ruch der Fiktion steht. 
In dieser Hinsicht scheint mir Joseph Vogls Formel von der Poetologie des Wissens $^{31}$ besonders aufschlußreich für die Kulturwissenschaft im Allgemeinen zu sein, verbindet sie doch in signifikanter Weise die beiden von uns diagnostizierten Tendenzen der Entgrenzung und Entsubstantialisierung.

Der Begriff einer Poetologie des Wissens lehnt sich, wie kaum zu übersehen ist und wohl auch nicht übersehen werden soll, an Stephen Greenblatts Konzept einer poetics of culture ${ }^{32}$ an. Aber er unterscheidet sich zugleich von seinem Modell. Die Poetologie des Wissens verschiebt gegenüber einer Poetik der Kultur das Dichterische vom Gegenstand auf den epistemischen Umgang mit diesem Gegenstand. Nicht die Kultur selbst, sondern das Wissen von ihr ist poetisch. Ganz konsequent auf der Grundlage der zitierten Prämissen gedacht, läßt sich dieser Unterschied freilich nicht mehr so recht begründen.

Dabei wandelt sich allerdings auch der Begriff des Poetischen selbst nicht unerheblich. Bei Greenblatt ist sein Akzent auf die poiesis im etymologischen Sinne gelegt, auf das Machen und Gestalten als das tertium comparationis von Dichtung und Kultur. Diese Gleichschaltung beider Operationen schafft nicht zuletzt die Voraussetzung dafür, um sie im hermeneutischen Umgang mit dem literarischen Text kurzzuschließen und die Literatur selbst als einen Seismographen historischer kultureller (und sozialer) Konstellationen zu deuten.

Dieser seiner Natur nach hermeneutische Zugriff auf den literarischen Text aber ist als solcher im New Historicism zum Verschwinden gebracht. Verantwortlich dafür ist ein bestimmtes terminologisches Kunststück, das sich mit dem allseits Verwendung findenden, überhaupt ziemlich beliebt gewordenen Begriff der negotiations verbindet. Vermutlich verdankt sich seine Popularität nicht zuletzt seiner Unschärfe. Denn was eigentlich besagt die Metapher der negotiations $^{33}$, der unweigerlich metaphorisch werdenden Verhandlungen, wenn sie denn nicht eine faktische Gesprächssituation zum Inhalt haben?

In methodologischer Hinsicht stellen diese negotiations indessen die auf den Gegenstand der Interpretation, nämlich den literarischen Text selbst, verschobenen Operationen einer literarischen Hermeneutik dar. Die negotiations erklären den Text selbst zum Ort dessen, was de facto ein Interpret betreibt. Rhetorisch läßt sich auf diese Weise der unbeliebten Hermeneutik womöglich

31 Vgl. Joseph Vogl: Robuste und idiosynkratische Theorie. In: KulturPoetik 7 (2007), S. 249258, hier S. 254.

32 Vgl. Stephen Greenblatt: Introduction. In: Ders.: The Forms of Power and the Power of Forms in the Renaissance (Genre 15 [1982]), S. 3-6, hier S. 6.

33 Stephen Greenblatt: Shakespearean Negotiations. The Circulation of Social Energy in Renaissance England. Berkeley, Los Angeles 1988. 
ein Mäntelchen umhängen; bei näherem Zusehen aber stammt es vermutlich aus der Werkstatt, in der auch der König seine neuen Kleider schneidern läßt.

Aber kommen wir zu Vogls Poetologie des Wissens zurück. Sie legt im Unterschied zu Greenblatts poetics of culture als eine Poetologie den Akzent nicht auf das Gemachte, auf das Gestaltende der poiesis, sondern auf den wahrheitssemantischen Status der Rede über die Dinge, anders gesagt auf den Wahrheitswert des Diskurses. Damit tritt ein zweites Modell von Vogls terminologischer Formel in den Blick, nämlich Michel Foucaults Archéologie du savoir, seine Archäologie des Wissens. ${ }^{34}$ Aber auch von diesem Modell unterscheidet sich die Poetologie des Wissens wiederum. Was aber ändert sich im Wechsel von der Archäologie zur Poetologie?

Die Archäologie in ihrer gewohnten disziplinären Form ist an der Rekonstruktion der in der Tiefe versteckten Fundamente interessiert. Eben dies ist in gewohnt metaphorischer Transposition auch das Anliegen Foucaults, möchte er doch die allen tatsächlichen Äußerungen zugrunde liegenden Regeln, die sie allererst ermöglichen und ihre Verfaßtheit determinieren, ans Tageslicht bringen.

Diese Eigenheit von Foucaults Konzept einer Archäologie des Wissens wirft im Übrigen eine Frage im Blick auf sein drei Jahre zuvor erschienenes Werk Les Mots et les choses, (Die Ordnung der Dinge) auf. ${ }^{35}$ Sie liegt umso näher, als der Untertitel dieses Buches ja bereits auf die Archéologie du savoir vorausdeutet, beide Bände also in einem unverkennbaren sachlichen Zusammenhang stehen. Das Problem, das sich im Blick auf die Beziehung zwischen ihnen stellt, betrifft die historische Reichweite, die eine Archäologie des Wissens unter den Voraussetzungen einer Konzeption von Wissensordnungen besitzen kann, wie sie in Les Mots et les choses entwickelt wird. Geht Foucault dort nämlich von radikalen Brüchen zwischen den einzelnen epistemologischen Formationen, die ohne einen erkennbaren Zusammenhang in historischer Reihe aufeinander folgen, aus, so erscheint es alles andere als evident, daß sie sich auch einem gleichen Konzept des Wissens fügen, wie es mit einer Unterscheidung zwischen Tiefe und Oberfläche zweifellos vorgezeichnet wird. Denn eben dieser Gegensatz macht ja gleichsam eine Prämisse aller archäologischen Tätigkeit aus. Er weist denn auch eine bemerkenswerte Ähnlichkeit mit einer der epistemai, die Foucault entwirft, auf: mit der sog. Tiefenepisteme der Moderne, die die Ordnung der Repräsentation ablöst, welche für das (aus französischer Sicht) klassische Zeitalter kennzeichnend war:

34 Michel Foucault: L’Archéologie du savoir. Paris 1969. (Deutsche Übersetzung: Archäologie des Wissens. Frankfurt a.M. 1984).

35 Michel Foucault: Les Mots et les choses. Une archéologie des sciences humaines. Paris 1966 (deutsche Übersetzung: Die Ordnung der Dinge. Frankfurt a.M. 1974). 
Désormais le tableau, cessant d'être le lieu de tous les ordres possibles, la matrice de tous les rapports, la forme de distribution de tous les êtres en leur individualité singulière, ne forme plus pour le savoir qu'une mince pellicule de surface; [...]. L'ordre qui se donne au regard, avec le quadrillage permanent de ses distinctions, n'est plus qu'un scintillement superficiel au-dessus d'une profondeur. ${ }^{36}$

Wäre das Konzept einer `Archäologie des Wissens` also insgeheim ein Produkt der Episteme der Moderne, die sich in diesem theoretischen Modell selbst spiegelte ihre Pertinenz gleichsam durch einen Anspruch historischer Universalität zu befestigen trachtete?

Die Zielrichtung einer Poetologie des Wissens weist stattdessen im Grunde in die genau entgegengesetzte Richtung von Foucaults Archäologie des Wissens. Auch für sie spielen die Konstruktionsregeln zweifellos eine nicht unwesentliche Rolle, aber sie setzt den Akzent auf die Konstruiertheit, das Poetische meint aus ihrer Sicht vor allem das Fiktive. Während Foucault agnostisch bleibt gegenüber dem ontologischen Status der Phänomene, die er beschreibt, richtet Vogl sein Augenmerk gerade auf ihren fiktiven Charakter. Seine Ausführungen zur Finanzwelt geben darüber beredte Auskunft. In seiner Studie Das Gespenst des Kapitals heißt es:

Die Kreditzirkulation basiert auf der Paradoxie eines, sich selbst garantierenden Geldes' und erweist sich als Schauplatz effektiver Fiktionen oder ,Dichtung', auf dem der Umlauf des Scheinhaften tatsächlich zur Determinante ökonomischer Relationen gerät. ${ }^{37}$

Ich will mich auf Vogls Einlassungen zur Finanzwirtschaft meinerseits nicht weiter einlassen, und dies schon allein deshalb nicht, weil ich mir für meinen Teil ziemlich sicher bin, davon nicht viel zu verstehen. Allerdings kann man sich des Eindrucks nicht erwehren, daß die zitierten Worte zwischen einer Theorie des Kapitals und einer Theorie des Romans oszillieren. Und sprach nicht schon Greenblatt im Blick auf die Literatur von einer circulation of social energy? Triebe hier am Ende womöglich eine Poetologie des Wissens ihren finanzpolitischen Zins ein? Aber wie immer es bei dieser Poetologie der Ökonomie um die Zuständigkeiten stehen mag, an Vogls Poetologie des Wissens läßt sich paradigmatisch der Unterschied zwischen einer Diskursarchäologie und der Kulturwissenschaft ablesen.

36 Foucault: Les Mots et les choses (s. Anm 35), S. 199. [»Von nun an bildet das tableau, das aufhört der Ort aller möglichen Ordnungen, die Matrix aller Beziehungen und die Form der Verteilung aller Wesen in ihrer besonderen Individualität zu sein, für das Wissen nur noch einen dünnen Film an seiner Oberfläche; $\{$... $\}$. Die Ordnung, die sich mit einer dauerhaften Einteilung ihrer Unterscheidungen dem Blick darbietet, ist nur noch ein oberflächliches Schillern über einer Tiefe.«] .

37 Joseph Vogl: Das Gespenst des Kapitals. Zürich 2010, S. 81. 
Auch Foucaults Diskurshistorie ist zweifelsohne nicht aus dem Taufbad der Metaphysik gehoben. Doch sein ontologischer Agnostizismus äußert sich in der kontingenten Abfolge unterschiedlicher historischer Formationen der Konditionierung aller Rede und Erkenntnis. Gerade das Bruchhafte des Übergangs von einer Formation zur nächsten erweist sich dabei als das Symptom ihrer metaphysischen Grundlosigkeit. ${ }^{38}$ Vermutlich hat Foucault dabei sogar ein wenig übertrieben. Es bedarf keiner sonderlichen Mühe, um die epochale Gliederung in Les Mots et les choses als den Spiegel einer Periodisierung der überkommenen Philosophiegeschichte zu lesen.

Die erste der beiden epistemologischen Schwellen, die Ablösung des Zeitalters der Ähnlichkeiten durch die Ordnung der Repräsentation, fällt ziemlich genau mit dem Aufkommen der cartesianischen Skepsis zusammen. Die Méditations métaphysiques erscheinen in lateinischer Sprache erstmals 1641. Für sie aber läßt sich durchaus nicht sagen, daß sie keine Kontinuität gegenüber dem vorausgehenden Denken aufweisen, wiewohl sie sich als ein absoluter Neuanfang begreifen - eine Diagnose, die in der Rezeption dieser Schrift durchaus bestätigt worden ist. Gleichwohl ergibt sich der Ansatz des radikalen Zweifels von René Descartes durchaus schlüssig aus der Entwicklung des europäischen Denkens. Die Gemeinsamkeit zwischen den Méditations métaphysiques und dem Ende des Zeitalters der Ähnlichkeiten besteht im Abbau des ontologischen Fundaments von Denken und Sprechen. Denn eben dies besagt es, daß mit der Ablösung der Ähnlichkeiten durch die Repräsentation das Band zwischen den Zeichen und der Welt aufgelöst wird, daß zwischen Zeichen und Bezeichnetem keine andere als die (bloße) Funktion der Bezeichnung selbst existiert, hingegen nicht mehr irgendeine Eigenschaft, die sie verbände (und damit motivierte), zu bemerken ist. Ein solcher Befund aber führt unweigerlich dazu, daß Sprechen und Denken auf sich selbst gestellt erscheinen, daß sie keine Garantie mehr für ihre Wahrheit in sich selbst tragen und so eine zutiefst nominalistische episteme auf den Weg bringen.

38 Foucault insistiert seit dem Vorwort von Les Mots et les choses auf dem bruchartigen Charakter der beiden epistemologischen Wechsel, die er um 1650 und um 1800 stattfinden läßt: »Or, cette enquête archéologique a montré deux grandes discontinuités dans l'épistémè de la culture occidentale: celle qui inaugure l'âge classique (vers le milieu du XVIIe siècle) et celle qui, au début du XIXe marque le seuil de notre modernité« (Foucault: Les Mots et les choses (s. Anm. 35), S. 9). [»Nun hat unsere archäologische Untersuchung zwei große Diskontinuitäten in der Episteme der westlichen Kultur aufgezeigt: diejenige, die \{gegen Mitte des 17. Jahrhunderts\} und diejenige, die am Beginn des 19. Jahrhunderts die Schwelle zu unserer Moderne einleitet.«]. 
Die Schlüssigkeit dieses Perspektivwechsels aber ergibt sich aus dem Ausfall einer als solcher unbefragten Prämisse des mittelalterlichen Denkens: aus dem Wegfall der theoretischen Leistung der Instanz Gottes. Galt der Schöpfer des Universums etwa ein Jahrtausend lang als Ursprung und Garant allen Seins, so verliert er diese Position am Beginn der Neuzeit. Sein Rollenwechsel tritt deutlich bei Descartes zutage: Weil die Selbstreflexivität des Cogito ergo sum keine Gewißheit für die Möglichkeit einer Erkenntnis der dem Denken des Ichs äußerlichen Gegenstände bietet, greift Descartes noch einmal auf den transzendenten Gott zurück, dessen Vollkommenheit es ausschließt, daß er seine Geschöpfe mit täuschenden Erkenntnismitteln ausgestattet habe. Diese Sicherung der Gegenstandserkenntnis aber bedarf nun einer aufwendigen Begründung prekärer Plausibilität, weshalb das neuzeitliche Denken sich von ihr auch bald distanziert hat. Die Existenz Gottes aber hat sich mithin bei Descartes vor der Logik zu rechtfertigen, weil dieser Gott nicht mehr umgekehrt als selbstverständlicher Begründer von Logos und Sein fungiert. Gott und Logos fallen auseinander. Der einstige Begründer des Logos bedarf nun selbst einer rationalen Rechtfertigung seiner Existenz.

Gerade an diesem Seitenwechsel der Rolle Gottes wird ersichtlich, daß die Absage an die metaphysische Deckung von Denken und Sprechen durchaus ihre Logik besitzt. Die Ablösung der Ähnlichkeit durch die Repräsentation bildet insofern die diskursgeschichtliche Facette einer allgemeinen Entwicklung des europäischen Denkens auf dem Weg in die Neuzeit, der es durchaus nicht an einer gewissen Schlüssigkeit mangelt. Ihr den Charakter einer bloßen, sich jeder Erklärung entziehenden discontinuité zuzusprechen, kann darum nicht wirklich überzeugen.

Ähnliches gilt für den zweiten epistemologischen Wechsel, den Foucault beschreibt: die Ablösung der Repräsentation durch die Tiefenepisteme der Moderne. Sie läßt sich durchaus konsequent als ein Effekt jener Selbstkritik der Vernunft beschreiben, die gegen Ende der Aufklärung einsetzt und die paradigmatisch im Werk Immanuel Kants zu Wort kommt.

Strukturell betrachtet sind die beiden epistemologischen Veränderungen übrigens von recht unterschiedlicher Natur. Während die alteuropäische, bis zur Renaissance Geltung besitzende Episteme auf der Ähnlichkeit beruht, rückt das Zeitalter der Repräsentation für Foucault stattdessen ihr Gegenteil, die Differenz, ins Zentrum einer diskursiven Ordnung, die hinfort wesentlich durch die differentiellen Merkmale eines tableau begründet wird. Die sich um 1800 ereignende Veränderung ist insofern von anderer Art, als die diskursbegründende Zeichenkonfiguration nicht durch einen Wechsel zum logischen Gegenteil zustande kommt, sondern durch die Integration der alten Zeichenordnung in eine neue erfolgt, wobei diese Ergänzung mit einer Entmächtigung ein- 
hergeht: Das tableau der Klassik sinkt herab zur bloßen Oberfläche einer Tiefe, in der sich die bestimmenden Kräfte des Seins regen. ${ }^{39}$

Genau dies aber entspricht den Konsequenzen der »Kopernikanischen Wende« Kants - und vor allem denen, die die Philosophie des 19. Jahrhunderts daraus gezogen hat. Man denke nur an Schopenhauers Metaphysik und ihre Umbesetzung des »Dings an sich« zum »Willen«, während Kants »Erscheinung « zur bloßen »Vorstellung « herabsinkt. Doch wäre es abwegig, der kantischen Wende selbst ihre Schlüssigkeit abzusprechen. Sie folgt sehr schlüssig aus einer aufklärerischen Anwendung der Vernunftkritik auf diese selbst. Die von Foucault reklamierten Eigenschaften der rupture und discontinuité treffen insoweit kaum zu. Aber just diese Diskrepanz zeigt ihre Funktion an, die es nun zu erörtern gilt.

Die Geschichte scheint in Les mots et les choses noch immer - gleichsam als ein Hegelianischer Rest e negativo - metaphysisch verdächtig zu sein. Zumal ihre Kontinuität noch immer zu suggerieren scheint, daß sie mehr als chronologischer Natur ist. Deshalb muß die radikale Kontingenz des Übergangs von einer Episteme zur nächsten her, um allen Fallstricken der Ontologie zu entkommen. Allerdings - und hier zeigt sich ein entscheidender Unterschied zur Kulturwissenschaft - bestimmt diese Abwehr aller Metaphysik nicht die Merkmale einer jeden epochalen Konfiguration. Die Ähnlichkeit, das Prinzip der Repräsentation oder die Tiefenepisteme, wie sie als wechselnde epochale Signaturen allen Sprechens und Denkens in Les Mots et les choses beschrieben werden, sind nicht notwendigerweise aufgrund ihrer Merkmalsbestimmtheit, sondern nur in ihrem Status von Foucaults elementarer ontologischer Skepsis geprägt. Sie unterscheiden sich, was ihre jeweiligen Eigenschaften angeht, in ontologischer Hinsicht durchaus voneinander: Die Ähnlichkeit verbindet die Zeichen mit den Dingen, die Repräsentation löst ihren sachlichen Zusammenhang auf, die Tiefenepisteme restituiert ihn als einen spekulativen Verbund. Die jeweiligen Regeln, die den Diskurs bestimmen, sind zugleich von so abstrakter Natur, daß sie einzelnen Gegenstandsbereichen durchaus ihre relative objektspezifische Autonomie belassen.

An genau diesem Punkt scheint mir der wesentliche Unterschied zwischen Kulturwissenschaft und Diskursarchäologie zutage zu treten. Die sprachzentrierte Kulturwissenschaft greift auf ein bestimmtes Medium zurück, eben die Sprache, der eine doppelte Aufgabe zufällt: Sie bietet ein Modell für die Strukturierung der Dinge an, die nach dem Vorbild sprachlicher Muster vermessen werden; und sie hat zugleich die Abwehr metaphysischer Ansprüche

39 Vgl. das Zitat auf S. 52 dieses Aufsatzes. 
zu sichern. Nicht zuletzt von hierher stammt die oben diskutierte, für die Kulturwissenschaft charakteristische, inflationäre Beteuerung von Selbstreferenzen aller Art.

Die Diskursarchäologie definiert stattdessen Konditionierungen des Denkens und Sprechens, die der Sprache vorausliegen, auch wenn sie sich in sprachlichen Entitäten wie bestimmten Zeichenkonzeptionen materialisieren. Nirgends wird dies vielleicht so deutlich wie in Foucaults Bestimmung der Tiefenepisteme der Moderne, für die er drei Transzendentalien ansetzt: das Leben, die Arbeit und eben die Sprache. ${ }^{40}$ Kulturwissenschaft und Diskursarchäologie teilen eine dezidiert anti-substantialistische Position für den Umgang mit kulturellen Phänomenen. Sie unterscheiden sich indessen maßgeblich in der Art und Weise, wie diese ontologische Skepsis jeweils zum Tragen kommt.

Die Diskursarchäologie, die sich als eine Diskurshistorie präsentiert, sichert die Autonomie gegenüber allen substantialistischen Ansprüchen durch eine radikale Historisierung von Formationsregeln für den Diskurs. Die Kulturwissenschaft überträgt diese Aufgabe stattdessen der Sprache selbst, die als das Medium aller Wirklichkeitskonstitution fungiert. In der Konsequenz dieser ihr zugedachten Funktion werden die Eigenschaften der Dinge ununterscheidbar von ihrer sprachlichen Charakteristik und damit haben sie notwendigerweise keinen anderen Grund als ihre Beschreibung mit den Mitteln der Sprache.

Die literaturwissenschaftliche Kulturwissenschaft steht deshalb Derrida deutlich näher als Foucault. Sie exekutiert gleichsam Derridas Postulat il n'y a

\footnotetext{
40 Die Beziehung der foucaultschen Diskursarchäologie zum linguistic turn erweist sich insofern als ein durchaus komplexes Verhältnis. Eine Affinität zu dessen Konsequenzen scheint darin zu bestehen, daß die drei epistemologischen Ordnungen, die Foucault in Les Mots et les choses entwirft, die Ordnungen der Ähnlichkeiten, der Repräsentation und der Tiefe, sich jeweils an einem bestimmten Verständnis des (sprachlichen) Zeichens ausrichten. Aber schon ihre als kontingent betrachtete Variation deutet an, daß die Geltung der jeweiligen Zeichenkonzeption sich nicht aus der Sprache und ihrem Wesen selbst heraus ableiten läßt. In diesem Sinn ist in der Tat das Zeichen der Tiefenepisteme besonders sprechend, weil es sich sozusagen selbst bereits in seiner Begrenztheit darbietet und den Blick auf Voraussetzungen des Denkens öffnet, die sich seiner Reichweite entziehen. Womöglich liegt deshalb in Foucaults Konzept einer Archäologie des Wissens auch schon der Ansatz zu jener Wende seines Denkens, die es mit und seit seiner Antrittsrede am Collège de France nehmen wird und die man als den Wechsel von der Archäologie zur Genealogie beschrieben hat (vgl. etwa Angèle Kremer-Marietti: Michel Foucault. Archéologie et généalogie. Paris 1985). Dem sprachlichen Zeichen und den Regeln ihres Gebrauchs liegt letztlich schon in Les Mots et les choses eine dort nicht reflektierte Konditionierung voraus, die im Postulat der schieren discontinuité als eine solche eher versteckt als thematisiert wird, deren Identität sich Foucault in seinem späteren Werk jedoch intensiv widmen und die er vor allem in der Kategorie einer anonymen Macht entdecken wird.
} 
pas de hors-texte ${ }^{41}$ indem sie an anderen als sprachlichen Objekten die Prinzipien von dessen Sprachanalyse zur Anwendung bringt. So sehr sich die literaturwissenschaftliche Kulturwissenschaft ihrem forschungsgeschichtlichen Selbstverständnis nach als eine Ablösung des Immanentismus der Dekonstruktion versteht, sie bleibt dessen konzeptuellen Prinzipien in beträchtlichem Maße verhaftet; nur dehnt sie deren Verfahren auf andere als nur sprachliche Phänomene aus.

Kommen wir indessen noch einmal auf die Gender Studies zurück, an denen sich noch ein weiteres Kennzeichen der Kulturwissenschaft beobachten läßt. Wenn für die Geschlechterforschung ein wesentliches Anliegen in der Demonstration, wo nicht Entlarvung der vermeintlichen Natürlichkeit von kulturell (und vor allem diskursiv) begründeten Geschlechterkonstruktionen besteht, so ist damit nämlich nur die eine Hälfte ihrer Zielvorgaben angesprochen. Der Feldzug gegen alle vermeintlichen Substantialismen geht dort auch mit einem besonderen Interesse für den Körper, für den aus aller kulturellen Überformung entlassenen, ja befreiten Körper, also dem Interesse für die bloße Körperlichkeit einher. Im Falle der Geschlechterforschung artikuliert sich dieses Anliegen vorzugsweise im Zeichen eines emanzipatorischen Programms, der Zurückweisung aller sozioethischen Restriktionen des Umgangs mit dem Körper.

Doch die Fokussierung auf den Körper ist keineswegs den besonderen Umständen der Gender Studies geschuldet. Sie bildet eine generelle Tendenz der Kulturwissenschaft. Signifikant in dieser Hinsicht ist, daß sie sich auch dort geltend

41 Jacques Derrida: De la grammatologie. Paris 1967, S. 220. Der Kontext dieses Satzes gibt zu erkennen, wie die Universalisierung der sprachlichen Verfaßtheit aller Gegenstände und ihre ontologische Grundlosigkeit auch in Derridas vielzitiertem Diktum unmittelbar miteinander zusammenhängen: »Et pourtant, si la lecture ne doit pas se contenter de redoubler le texte, elle ne peut légitimement transgresser le texte vers autre chose que lui, vers un référent (réalité métaphysique, historique, psycho-biographique, etc.) ou vers un signifié hors texte dont le contenu pourrait avoir lieu, aurait pu avoir lieu hors de la langue, c'est-à-dire, au sens que nous donnons ici à ce mot, hors de l'écriture en général. C'est pourquoi les considérations méthodologiques que nous risquons ici sur un exemple sont étroitement dépendantes des propositions générales que nous avons élaborées plus haut, quant à l'absence du référent ou du signifié transcendantal. Il n’y a pas de hors-texte« (ibid.). [»Und dennoch, wenn die Lektüre sich nicht damit begnügen soll, den Text zu doppeln, kann sie ihn legitimerweise zu etwas anderem als ihm selbst auch nicht überschreiten, zu einem Referenten (einer metaphysischen, historischen oder psycho-biographischen etc. Wirklichkeit) oder zu einem Signifikat außerhalb des Textes, dessen Inhalt sich ereignen könnte, sich außerhalb der Sprache hätte ereignen können, d. h. außerhalb der - in dem Sinn, den wir diesem Wort hier geben - écriture im Allgemeinen. «]. Deshalb hängen die methodologischen Erörterungen, die wir hier an einem Beispiel erproben, unmittelbar von den allgemeinen Aussagen, die wir oben bezüglich der Abwesenheit des Referenten oder des transzendentalen Signifikats entwickelt haben, ab. Es gibt kein Außerhalb des Textes. 
macht, wo man es nicht unbedingt erwarten würde. Ich erwähne als Beispiel nur die Arbeiten der Philosophin Sybille Krämer, die in einer ganzen Reihe von Studien den Belang des Körperlichen für die Sprache herausgestrichen hat und Deixis wie Metapher auf körperliche Phänomene zurückführen möchte. ${ }^{42}$

Daß eine kritische, wo nicht radikal skeptische Position gegenüber der Substanz alles Intelligiblen oder Konzeptuellen der anderen Seite dieser uralten Dichotomie ein größeres Gewicht zusprechen möchte, liegt auf der Hand. Das signifiant gewinnt gegenüber dem signifié unter solchen Voraussetzungen unweigerlich an Gewicht, ja die Oberhand. Und so kommt es zu den vielzitierten sfrei flottierenden Signifikanten`. Doch gerade an dieser Formulierung zeigt sich das Dilemma einer solchen mission impossible. Denn wenn es so wäre, daß sich in ihnen das Körperliche in seinem Eigenrecht behaupte, ist doch zum anderen nicht zu übersehen, daß es als ein solches im Grunde gar nicht zum Vorschein kommt. Genau genommen, ist deshalb schon der Begriff der frei flottierenden Signifikanten irreführend. Denn das, was flottiert, sind die Signifikate. Sie - und nicht etwa deren körperliche Träger - sind instabil. Körperlichkeit aber wird auf diese Weise zum bloßen Effekt einer Aufhebung von stabiler Semantik. Der Körper bleibt im Grunde das Andere des Intelligiblen, dem keine weitere Bestimmtheit als dessen (bloße) Negation zukommt.

Und damit sind wir auch schon beim Kern des Problems dieser quête $d u$ corps angekommen. Wo immer der Diskurs sich des Körpers zu bemächtigen bemüht, macht er es nach dem Gesetz, nach dem er angetreten. Er kann es nicht anders als vermittels eines Bezugs zur Semantik tun. Noch der Dadaismus fußt auf diesem Prinzip. Denn erst die Abwesenheit von Sinn macht den Laut-Körper belang-, um nicht zu sagen: bedeutungsvoll. Angemessener wäre es wohl zu sagen: bedeutsam. Auch in einer solchen poetischen Praxis tritt der Körper (erst) im Modus der Negation von Sinn in den Fokus der Aufmerksamkeit. Übrigens läßt sich gerade anhand des Dadaismus die tiefe Verwurzelung der Semantik in der Sprache studieren. Während das abstrakte Bild seinen Siegeszug in der Kunst der Moderne angetreten hat, ist dadaistische Dichtung kaum mehr als eine Episode geblieben.

Das Faszinosum, ja das Phantasma des Körperlichen in der Kulturwissenschaft erweist sich solchermaßen als eine Wirkung von dessen Unerreichbarkeit. Das Unzulängliche aber wird - auch mit den ihr zur Verfügung stehenden Mitteln -

42 Vgl. etwa: Sybille Krämer: Sagen und Zeigen. Sechs Perspektiven, in denen das Diskursive und das Ikonische in der Sprache konvergieren. In: Zeitschrift für Germanistik N.F. 3 (2003), S. 509-519. Ich will an dieser Stelle gegen ihre These einer grundsätzlichen Affinität von Metaphorik und Körperlichkeit nur eine durchaus geläufige Metapher nennen, in der diese Beziehung kaum eine Rolle spielt: `etwas auf einen Nenner bringen`. 
gerade nicht Ereignis. ${ }^{43}$ Zum lieu de désir der Moderne gerät der Körper vielmehr als ein Quell einer elementaren Paradoxie ihres Denkens.

Vielleicht nirgends deutlicher als in Kants Erkenntnistheorie kommt dessen basales Paradoxon zum Vorschein. Das Körperliche, das mit den Sinnen Erfaßbare, markiert die Grenze der Erkenntnisfähigkeit des Menschen. Doch dieses Körperliche wird der Vernunft nur auf dem Umweg der Kategorien, die sie selbst vorgibt, zugänglich. Es bleibt insofern die große Leerstelle dieser epistemologischen Konstellation. Der Vernunft ihre Grenze setzend, bleibt der Körper derselben Vernunft doch entzogen. So gerät der Körper letztlich in die Rolle eines, wo nicht des Dings an sich, um noch einmal einen Begriff der Kritik der reinen Vernunft zu verwenden. Hier scheint mir die Ursache seines immensen wissenschaftlichen Attraktionspotentials zu liegen, dessen Intensität mit dem bloßen Aufstand gegen alle sozialen sstaatlichen oder religiösen Restriktionen von Körperlichkeit trotz seines Freiheitspathos` keine rechte Plausibilität gewinnt.

Auch die kulturwissenschaftliche Fixierung auf den Körper gründet mithin auf der benannten epistemologischen Paradoxie, aber sie gründet darin im doppelten Sinne des Wortes. Denn sie verkennt, daß sie auf diese Weise selbst zu einer paradoxen Unternehmung gerät. Sie ist im Grunde nicht die Lösung, sondern ein Symptom der Verwerfungen des Denkens der Moderne.

Nun ist es, wie dem ausführlichen und sehr pointierten Prospekt des Kolloquiums, dem dieser Beitrag entstammt, zu entnehmen ist, sein erklärtes Anliegen die Erkundung der Möglichkeiten einer Neubegründung von Sozial- und Ideengeschichte der Literatur. So verlockend ein solches Vorhaben angesichts der nicht zu leugnenden Blindstellen einer literaturwissenschaftlichen Kulturwissenschaft erscheint, gilt es doch der Gefahr zu wehren, daß auch die ins Auge gefaßte Reorientierung als nichts anderes denn ein weiteres Glied in der schier endlosen Kette immer neuer turns endet. Um dies zu vermeiden, sollten m. E. vor allem zwei Voraussetzungen erfüllt sein:

43 Sprechend in dieser Hinsicht scheint mir übrigens Judith Butlers intelligente Wendung zu sein, die sie der Frage nach der Körperlichkeit des Geschlechts gibt. Nachdem sie hellsichtig die Widersprüche aufgezeigt hat, die der herkömmliche Konstruktivismus der Geschlechterforschung in seinen verschiedenen Varianten für die Unterscheidung zwischen sex und gender mit sich bringt, schlägt sie als Lösung vor, die materiality of sex wie folgt in die Perspektive zu rücken: »Thus, the question is no longer, How is gender constituted as and through a certain interpretation of sex? (a question that leaves the 'matter' of sex untheorized), but rather, Through what regulatory norms is sex itself materialized?« (Butler: Bodies that Matter [s. Anm. 30], S. 10). 
1) Perspektiven einer neuen Ideen- und Sozialgeschichte der Literatur - um den Untertitel unseres Symposiums zu zitieren - wird es nur geben können, wenn sie sich auch vom Genitiv »der Literatur« in diesem Programm zu lösen vermag. Erfolg wird sie nämlich dauerhaft nur gewinnen können, wenn sie sich ebenso als literarische Ideen- und Sozialgeschichte zu etablieren vermag. Hinter diesem grammatischen Postulat verbirgt sich durchaus mehr als nur rhetorisches Paradeexerzieren. Gemeint ist damit die Notwendigkeit, den medialen Belang einer solchen Geschichte aus den konstitutiven Eigenheiten der Literatur selbst zu begründen. Und dies wird nur in einer doppelten Perspektive gelingen. Zum einen gilt es, die Funktion der Literatur innerhalb einer Ideen- und Sozialgeschichte zu erforschen, in der die Literatur einen Teil bildet. Zum anderen gilt es umgekehrt, den Belang von Ideen- und Sozialgeschichte für die Literatur zu erweisen. Beide Perspektiven bedürfen vor allem ihrer Verknüpfung. Erst vermittels ihrer wechselseitigen Spiegelung werden sich tragbare Ergebnisse einstellen. Forschungsgeschichtlich gesprochen: Es muß das Kunststück her, Werkinterpretation und Literatursoziologie miteinander zu verkoppeln. Man könnte auch, ein wenig grundsätzlicher, sagen: Hermeneutik und Geschichte harren ihrer literaturwissenschaftlichen Versöhnung. Der New Historicism, dem ein solches Anliegen ja keineswegs fremd ist, hat diese Verbindung gleichwohl nicht wirklich geleistet. Er hat, wie bereits diskutiert, unter der Hand eine literarische Hermeneutik auf ihren Gegenstand verschoben und die Literatur als solche in das Medium einer solchen Vermittlung zwischen ihr selbst und ihrer kulturellen Umgebung verwandelt - als sei zwischen beidem gar nicht zu unterscheiden. Auch in diesem Sinne ist die Formel der poetics of culture im New Historicism sprechend: Wenn die Kultur nach dem Modell der Dichtung gebaut ist, dann deshalb, weil die Literatur ihrerseits die Kultur in den Zusammenhang bringt. Das - zutiefst metaphorische Zauberwort dafür heißt, wir haben es besprochen: negotiations. Auf diese Weise aber wird die Arbeit des Interpreten in dem von ihm bearbeiteten Gegenstand gleichsam aufgehoben (um nicht $\mathrm{zu}$ sagen: versteckt). Stattdessen gilt es, die wechselseitige Durchdringung von Literatur und (anderweitiger) Kultur nicht zum literarischen Verfahren als solchem zu erklären, sondern ihre wechselseitigen Korrelationen empirisch $\mathrm{zu}$ beobachten.

2) Eine Reorientierung, wie sie der Prospekt unseres Kolloquiums vorschlägt, schließt auch eine gründliche Auseinandersetzung mit den epistemologischen Voraussetzungen der Kulturwissenschaft ein. Und dazu gehört vor allem eine kritische Revision postmoderner Sprach- und Literaturtheorie. Wenn ich gerade ihren Belang so herausstelle, dann nicht nur, weil sie sich 
subkutan in vielen Ansätzen verbirgt, die vordergründig ganz anderen Zielsetzungen verpflichtet sind. Vielmehr ist diese latente Ubiquität nur ein Symptom der Ursache, die die postmoderne Theorie in der Literaturwissenschaft so populär macht.

Es ist in dieser Hinsicht äußerst aufschlußreich, daß die strukturale Theorie der poetischen Sprache in beträchtlichem Maße der poststrukturalen Theorie der Sprache entspricht. Roman Jakobsons poetische Funktion mit der für sie kennzeichnenden Autoreferentialität entspricht nämlich sehr weitgehend dem generellen Modell der Sprache, das der Poststrukturalismus entwickelt hat. Sie schafft im Grunde den Gegensatz zwischen Referentialität und Autoreferentialität (zugunsten letzterer) ab. Diese Affinität aber enthält einen latenten Hinweis auf die Gründe der Beliebtheit dekonstruktiver Theorie in der Literaturwissenschaft (und hält dabei zugleich eine Erklärung dafür bereit, warum sie hier - und nicht dort, wo es von ihrem Gegenstand her zu erwarten wäre, nämlich in Philosophie und Sprachwissenschaft ihren eigentlichen Erfolg gefeiert hat): Die Dekonstruktion bietet in letzter Konsequenz eine theoretische und terminologische Neuformulierung wesentlicher Aspekte romantischer Poetik. Der Vorteil, den sie bietet, ist die Fundamentierung dieser Poetik in einer Theorie der Sprache selbst.

Um nur einige Hinweise zur Begründung dieser These $\mathrm{zu}$ geben: Als sich die Philologien im 19. Jahrhundert konstituierten, bestimmte die romantische Konzeption der Literatur weithin die Vorstellung vom Wesen des Poetischen. Es ist dieses ihren Anfängen geschuldete Erbe, das die Literaturwissenschaft bis auf den heutigen Tag nachhaltig prägt.

Die romantische Poetik richtete sich bekanntlich gegen die Präzepte des in beträchtlichem Maße von der Rezeption der aristotelischen Poetik bestimmten Klassizismus, der vom 16.-18. Jahrhundert die dominante poetologische Referenz darstellte. Die Regeln klassizistischer Poetik beruhten auf rationalen Prinzipien: Klarheit, Wahrscheinlichkeit und Schlüssigkeit galten als Richtschnur literarischer Produktion. Die Poetik der Romantik gründet stattdessen wesentlich auf einer Rebellion gegen diese Prinzipien. Dieser Widerstand - und dies bildet eine wesentliche Voraussetzung romantischer Poetik - wird weithin angeregt durch das Denken der späten Aufklärung mit ihrer Selbstkritik der Vernunft, die vor allem die Grenzen dieser Vernunft aufgezeigt hat. So bringt Kants Denken die Einsicht mit sich, daß die unhintergehbare Rationalität des menschlichen Zugriffs auf die Welt keineswegs verbürgt, daß die Welt selbst nach rationalen Prinzipien organisiert ist. Auf diese Weise ergibt sich ein Hiat zwischen dem Denken und der Wirklichkeit, und genau dieser Lücke, die das aufklärerische Denken produziert hat, bemächtigt sich die Romantik. Sie versucht 
dort Einsichten zu gewinnen, wo rationale Verfahren an das Ende ihrer Möglichkeiten gelangen. Romantik ist insofern durchaus nicht, jedenfalls nicht nur eine Gegenbewegung gegen die Aufklärung, als welche man sie gern versteht: Sie ist zunächst ihre durchaus konsequente Folgeerscheinung.

Das hier skizzierte Verhältnis von Romantik und Aufklärung kann erklären, warum die romantische Poetik die Prinzipien des Klassizismus so grundsätzlich in Frage stellt. Nicht Wahrscheinlichkeit, Schlüssigkeit und rationale Transparenz bestimmen das poetische Werk, sondern gerade deren Gegenteil. Wenn der rationale Zugriff auf die Welt das Sein der Dinge verfehlt, eröffnet dann nicht vielleicht ihr Gegenteil den Zugang zu ihrem Wesen? Nicht Klarheit, sondern Vagheit, nicht Eindeutigkeit, sondern Vieldeutigkeit haben sich deshalb dort zu bewähren, wo ihr der Vernunft verpflichtetes Gegenprogramm scheitert. Das Mysteriöse und Unheimliche und nicht mehr das Schlüssige und Wahrscheinliche ist die Domäne romantischer Literatur.

Genau diesen anti-rationalistischen Zug aber teilt die Poetik der Romantik mit der Theorie der Dekonstruktion. Keine stabilen, auf logischen Beziehungen gegründete Bedeutungen sind aus post-strukturalistischer Sicht das Merkmal der Sprache, sondern semantische Dispersion, ein nie ans Ende kommender Prozeß des Aufschubs von Bedeutung macht ihr Wesen aus. Und diese Skepsis gegenüber aller Rationalität, den die Romantik mit dem Dekonstruktivismus teilt, macht sie so populär in der Literaturwissenschaft. Sie scheint eine avancierte Theorie für eine überkommene Position anzubieten und damit zugleich ein Dilemma zu lösen, das die Literaturwissenschaft vielleicht seit jeher umtreibt: den schwer auszuhaltenden Konflikt zwischen einem Gegenstand, dem weitgehend ein irrationaler Charakter zugesprochen wird, und den Ansprüchen einer unvermeidlich rationalen Unternehmung, als welche sich eine Wissenschaft nun einmal präsentiert.

Übrigens läßt sich auch eine gewisse Parallele in der Genese von Romantik und Dekonstruktivismus bemerken: So wie die Romantik aus der Selbstkritik der Aufklärung folgt, versteht sich auch Derridas Sprachtheorie als eine Fortentwicklung des Strukturalismus, dessen Blindstellen er beheben möchte. ${ }^{44}$

\footnotetext{
44 Diese Gemeinsamkeit gründet freilich weit mehr auf dem Anspruch, den der Post-Strukturalismus erhebt, als auf wirklich vergleichbaren Verhältnissen der Theorieentwicklung. Kants Kritik der reinen Vernunft artikuliert ein ontologisches Defizit der Vernunft, das seit Descartes das neuzeitliche Denken beschäftigt. Derrida muß hingegen, wie gesehen (vgl. S. 44ff.), Saussures Sprachtheorie und ihre Begriffe erheblich transformieren, um sie für seine ontologische Kritik allererst aufzubereiten.
} 
Der durchaus prekären Affinität von Literaturwissenschaft und Dekonstruktion aber wird man nur Herr werden können, wenn man die Grundlagen postmoderner Sprachtheorie kritisch befragt. Es führt mithin kein Weg vorbei an der Kritik der unreinen Vernunft, die sich in der postmodernen Sprachtheorie eingenistet hat.

Soll der Titel unserer Tagung - Nach der Kulturgeschichte - von einem Programm, ich will von Utopie ja gar nicht sprechen - zur Empirie wechseln, dann scheint mir eine kritische Auseinandersetzung mit den theoretischen Prämissen der Kulturwissenschaft unerläßlich zu sein. So begründet die Warnung vor der Entgrenzung des Literaturbegriffs ist und so berechtigt die Klage über den Verlust einer distinktiven Kompetenz erscheint, alle Rufe nach einer Rephilologisierung werden weitgehend wirkungslos verhallen, wenn dabei nur die Konsequenzen der Kulturwissenschaft kritisch in den Blick genommen werden, ihre theoretischen Voraussetzungen, die ihre Entstehung allererst zu erklären vermögen, aber unbefragt bleiben.

Deshalb gilt es, Kritik und Konstruktion miteinander zu verbinden und aus der Analyse der Defizite poststrukturaler Theorie den Ansatz für die Formulierung einer Alternative zu entwickeln. Übrigens scheint sich mir hier auch eine sinnvolle Zusammenarbeit zwischen Sprach- und Literaturwissenschaften abzuzeichnen, die sich zum wechselseitigen Schaden bis an die Grenze der Sprachlosigkeit voneinander entfernt haben. Ihre zerrüttete Ehe könnte in der gemeinsamen Diskussion postmoderner Sprachtheorie durchaus neuen Schwung bekommen. 
